\title{
Combinatorial complexity in o-minimal geometry
}

\author{
Saugata Basu
}

\begin{abstract}
In this paper we prove tight bounds on the combinatorial and topological complexity of sets defined in terms of $n$ definable sets belonging to some fixed definable family of sets in an ominimal structure. This generalizes the combinatorial parts of similar bounds known in the case of semi-algebraic and semi-Pfaffian sets, and as a result vastly increases the applicability of results on combinatorial and topological complexity of arrangements studied in discrete and computational geometry. As a sample application, we extend a Ramsey-type theorem due to Alon et al. 3, originally proved for semi-algebraic sets of fixed description complexity to this more general setting.
\end{abstract}

\section{Introduction}

Over the last twenty years there has been a lot of work on bounding the topological complexity (measured in terms of their Betti numbers) of several different classes of subsets of $\mathbb{R}^{k}$ - most notably semi-algebraic and semi-Pfaffian sets. The usual setting for proving these bounds is as follows. One considers a semi-algebraic (or semi-Pfaffian) set $S \subset \mathbb{R}^{k}$ defined by a Boolean formula whose atoms consists of $P>0, P=0, P<0, P \in \mathcal{P}$, where $\mathcal{P}$ is a set of polynomials (resp. Pfaffian functions) of degrees bounded by a parameter (resp. whose Pfaffian complexity is bounded by certain parameters) and $\# \mathcal{P}=n$. It is possible to obtain bounds on the Betti numbers of $S$ in terms of $n, k$ and the parameters bounding the complexity of the functions in $\mathcal{P}$.

\subsection{Known bounds in the semi-algebraic and semi-Pfaffian cases}

In the semi-algebraic case, if we assume that the degrees of the polynomials in $\mathcal{P}$ are bounded by $d$, and denoting by $b_{i}(S)$ the $i$-th Betti number of $S$, then it is shown in 21 that,

$$
\sum_{i \geq 0} b_{i}(S) \leq n^{2 k} O(d)^{k}
$$

A similar bound is also shown for semi-Pfaffian sets [21.

In another direction, we also have reasonably tight bounds on the sum of the Betti numbers of the realizations of all realizable sign conditions of the family $\mathcal{P}$. A sign condition on $\mathcal{P}$ is an element of $\{0,1,-1\}^{\mathcal{P}}$, and the realization of a sign condition $\sigma$ is the set,

$$
\mathcal{R}(\sigma)=\left\{\mathbf{x} \in \mathrm{R}^{k} \mid \operatorname{sign}(P(\mathbf{x}))=\sigma(P), \forall P \in \mathcal{P}\right\} .
$$

It is shown in 9 that,

$$
\sum_{\sigma \in\{0,1,-1\}^{\mathcal{P}}} b_{i}(\mathcal{R}(\sigma)) \leq \sum_{j=0}^{k-i}\left(\begin{array}{l}
n \\
j
\end{array}\right) 4^{j} d(2 d-1)^{k-1}=n^{k-i} O(d)^{k} .
$$

2000 Mathematics Subject Classification 52C45.

The author was supported in part by an NSF grant CCF-0634907. 
We refer the reader to $\mathbf{8}, \mathbf{9}, \mathbf{2 0}, \mathbf{2 1}, \mathbf{1 8}$, as well as the survey article 4 for a comprehensive history of the work leading up to the above results, as well as several other interesting results in this area.

\subsection{Combinatorial and algebraic complexity}

Notice that the bounds in (1.1) and (1.2) are products of two quantities - one that depends only on $n$ (and $k$ ), and another part which is independent of $n$, but depends on the parameters controlling the complexity of individual elements of $\mathcal{P}$ (such as degrees of polynomials in the semi-algebraic case, or the degrees and the length of the Pfaffian chain defining the functions in the Pfaffian case). It is customary to refer to the first part as the combinatorial part of the complexity, and the latter as the algebraic (or Pfaffian) part. Moreover, the algebraic or the Pfaffian parts of the bound depend on results whose proofs involve Morse theory (for instance, the well known Oleinik-Petrovsky-Thom-Milnor bounds on the Betti numbers of real varieties 25, 29, 24]).

While understanding the algebraic part of the complexity is a very important problem, in several applications, most notably in discrete and computational geometry, it is the combinatorial part of the complexity that is of primary interest (the algebraic part is assumed to be bounded by a constant). The motivation behind this point of view is the following. In problems in discrete and computational geometry, one typically encounters arrangements of a large number of objects in $\mathbb{R}^{k}$ (for some fixed $k$ ), where each object is of "bounded description complexity" (for example, defined by a polynomial inequality of degree bounded by a constant). Thus, it is the number of objects that constitutes the important parameter, and the algebraic complexity of the individual objects are thought of as small constants. It is this second setting that is our primary interest in this paper.

The main results of this paper generalize (combinatorial parts of) the bounds in (1.1) and (1.2) to sets which are definable in an arbitrary o-minimal structure over a real closed field $\mathrm{R}$ (see Section 1.4.1 below for the definition of an o-minimal structure and definable sets).

Instead of only considering sets having "bounded description complexity", we allow the sets in an arrangement $\mathcal{A}$ to be fibers of some fixed definable map $\pi: T \rightarrow \mathrm{R}^{\ell}$, where $T \subset \mathrm{R}^{k+\ell}$ is a definable set. This vastly expands the applicability of results concerning complexity of arrangements in discrete and computational geometry, since it is no longer necessary that the objects in the arrangements be defined only in terms of polynomials. As we will see shortly, the sets we consider are allowed to be fairly arbitrary. They include sets defined by restricted analytic functions, including (but not by any means restricted to) polynomials, Pfaffian functions such as exponential, logarithmic, trigonometric, inverse trigonometric functions, subject to some mild conditions. All hitherto considered families of objects in the computational geometry literature, such as hyperplanes, simplices, and more generally sets having bounded description complexity are special instances of this general definition. We also consider sets belonging to the Boolean algebra generated by $n$ sets in $\mathrm{R}^{k}$ each of which is a fiber of a fixed definable map. We prove tight bounds on the Betti numbers, the topological complexity of projections, as well as on the complexity of cylindrical decomposition of such sets, in terms of $n$ and $k$. The role of the algebraic complexity is played by a constant that depends only on the particular definable family. In this way, we are able to generalize the notion of combinatorial complexity to definable sets over an arbitrary o-minimal structure.

Apart from the intrinsic mathematical interest of the results proved in the paper, we believe that the techniques used to prove them would be of interest to researchers in discrete and computational geometry. We show that most (if not all) results on the complexity of arrangements are consequences of a set of very simple and well-studied axioms (those defining o-minimal structures). Many widely used techniques in the study of arrangements are strongly dependent on the assumption that the sets under consideration are semi-algebraic. For example, 
it is common to consider real algebraic varieties of fixed degree as hyperplane sections of the corresponding Veronese variety in a higher (but still fixed) dimensional space - a technique called "linearization" in computational geometry literature (see 2]). Obviously, such methods fail if the given sets are not semi-algebraic. Our methods make no use of semi-algebraicity of the objects, nor bounds derived from Morse theory such as the classical Oleinik-Petrovsky-ThomMilnor bounds on Betti numbers of real algebraic varieties. We believe that this point of view simplifies proofs, and simultaneously generalizes vastly the class of objects which are allowed, at the same time getting rid of unnecessary assumptions such as requiring the objects to be in general position. It is likely that the techniques developed here will find further applications in the combinatorial study of arrangements other than those discussed in this paper.

\subsection{Arrangements in computational geometry}

We now make precise the notions of arrangements, cells and their complexities, following their usual definitions in discrete and computational geometry [2, 22.

Let $\mathcal{A}=\left\{S_{1}, \ldots, S_{n}\right\}$, such that each $S_{i}$ is a subset of $\mathrm{R}^{k}$ belonging to some "simple" class of sets. (We will define the class of admissible sets that we consider precisely in Section 1.5 below).

For $I \subset\{1, \ldots, n\}$, we let $\mathcal{A}(I)$ denote the set

$$
\bigcap_{i \in I \subset[1 \ldots n]} S_{i} \cap \bigcap_{j \in[1 \ldots n] \backslash I} \mathrm{R}^{k} \backslash S_{j},
$$

and it is customary to call a connected component of $A(I)$ a cell of the arrangement (even though it might not be a cell in the sense of topology). We let $\mathcal{C}(\mathcal{A})$ denote the set of all non-empty cells of the arrangement $\mathcal{A}$.

The cardinality of $\mathcal{C}(\mathcal{A})$ is called the combinatorial complexity of the arrangement $\mathcal{A}$. Since different cells of an arrangement might differ topologically, it makes sense to give more weight to a topologically complicated cell than to a topologically simple one in the definition of complexity. With this in mind we define (following [7]) the topological complexity of a cell to be the sum of its Betti numbers (the ranks of singular homology groups of the cell).

The class of sets usually considered in the study of arrangements are sets with "bounded description complexity" (see [2]). This means that each set in the arrangement is defined by a first order formula in the language of ordered fields involving at most a constant number polynomials whose degrees are also bounded by a constant. Additionally, there is often a requirement that the sets be in "general position". The precise definition of "general position" varies with context, but often involves restrictions such as: the sets in the arrangements are smooth manifolds, intersecting transversally.

\subsection{Arrangements over an o-minimal structure}

O-minimal structures present a natural mathematical framework to state and prove results on the complexity of arrangements. In this paper we consider arrangements whose members come from some fixed definable family in an o-minimal structure (see below for definitions). The usual notion of "bounded description complexity" turns out to be a special case of this more general definition.

1.4.1. O-minimal structures O-minimal structures were invented and first studied by Pillay and Steinhorn in the pioneering papers [26, 27, in part to show that the tame topological properties exhibited by the class of semi-algebraic sets are consequences of a set of few simple axioms. Later the theory was further developed through contributions of other researchers, most notably van den Dries, Wilkie, Rolin, Speissegger amongst others [31, 32, 33, 34, 35, $\mathbf{2 8}$. We particularly recommend the book by van den Dries [30] and the notes by Coste $\mathbf{1 2}$ 
for an easy introduction to the topic as well as the proofs of the basic results that we use in this paper.

An o-minimal structure on a real closed field $\mathrm{R}$ is just a class of subsets of $\mathrm{R}^{k}, k \geq 0$, (called the definable sets in the structure) satisfying these axioms (see below). The class of semialgebraic sets is one obvious example of such a structure, but in fact there are much richer classes of sets which have been proved to be o-minimal (see below). For instance, subsets of $\mathbb{R}^{k}$ defined in terms inequalities involving not just polynomials, but also trigonometric and exponential functions on restricted domains have been proved to be o-minimal.

We now formally define o-minimal structures (following [12]).

Definition 1. An o-minimal structure on a real closed field $\mathrm{R}$ is a sequence $\mathcal{S}(\mathrm{R})=$ $\left(\mathcal{S}_{n}\right)_{n \in \mathbb{N}}$, where each $\mathcal{S}_{n}$ is a collection of subsets of $\mathrm{R}^{n}$, satisfying the following axioms [12.

(i) All algebraic subsets of $\mathrm{R}^{n}$ are in $\mathcal{S}_{n}$.

(ii) The class $\mathcal{S}_{n}$ is closed under complementation and finite unions and intersections.

(iii) If $A \in \mathcal{S}_{m}$ and $B \in \mathcal{S}_{n}$ then $A \times B \in \mathcal{S}_{m+n}$.

(iv) If $\pi: \mathrm{R}^{n+1} \rightarrow \mathrm{R}^{n}$ is the projection map on the first $n$ co-ordinates and $A \in \mathcal{S}_{n+1}$, then $\pi(A) \in \mathcal{S}_{n}$.

(v) The elements of $\mathcal{S}_{1}$ are precisely finite unions of points and intervals.

\subsubsection{Examples of o-minimal structures}

EXAMPLE 1. Our first example of an o-minimal structure $\mathcal{S}(\mathrm{R})$, is the o-minimal structure over a real closed field $\mathrm{R}$ where each $\mathcal{S}_{n}$ is the class of semi-algebraic subsets of $\mathrm{R}^{n}$. It follows easily from the Tarski-Seidenberg principle (see [1]) that the class of sets $\mathcal{S}_{n}$ satisfies the axioms in Definition 1 . We will denote this o-minimal structure by $\mathcal{S}_{\mathrm{sa}}(\mathrm{R})$.

If Example 1 was the only example of o-minimal structure available then the notion of ominimality would not be very interesting. However, there are many more examples (see for example [30, 31, 32, 33, 28, 34, 35).

EXAmple 2. [34] Let $\mathcal{S}_{n}$ be the images in $\mathbb{R}^{n}$ under the projection maps $\mathbb{R}^{n+k} \rightarrow \mathbb{R}^{n}$ of sets of the form $\left\{(\mathbf{x}, \mathbf{y}) \in \mathbb{R}^{n+k} \mid P\left(\mathbf{x}, \mathbf{y}, e^{\mathbf{x}}, e^{\mathbf{y}}\right)=0\right\}$, where $P$ is a real polynomial in $2(n+$ $k)$ variables, and $e^{\mathbf{x}}=\left(e^{x_{1}}, \ldots, e^{x_{n}}\right)$ and $e^{\mathbf{y}}=\left(e^{y_{1}}, \ldots, e^{y_{k}}\right)$. We will denote this o-minimal structure over $\mathbb{R}$ by $\mathcal{S}_{\exp }(\mathbb{R})$.

EXAMPLE 3. [19] Let $\mathcal{S}_{n}$ be the images in $\mathbb{R}^{n}$ under the projection maps $\mathbb{R}^{n+k} \rightarrow \mathbb{R}^{n}$ of sets of the form $\left\{(\mathbf{x}, \mathbf{y}) \in \mathbb{R}^{n+k} \mid P(\mathbf{x}, \mathbf{y})=0\right\}$, where $P$ is a restricted analytic function in $n+k$ variables. A restricted analytic function in $N$ variables is an analytic function defined on an open neighborhood of $[0,1]^{N}$ restricted to $[0,1]^{N}$ (and extended by 0 outside). We will denote this o-minimal structure over $\mathbb{R}$ by $\mathcal{S}_{\text {ana }}(\mathbb{R})$.

The o-minimality of the last two classes are highly non-trivial theorems.

\subsection{Admissible sets}

We now define the sets that will play the role of objects of "constant description complexity" in the rest of the paper. 
Definition 2. Let $\mathcal{S}(\mathrm{R})$ be an o-minimal structure on a real closed field $\mathrm{R}$ and let $T \subset \mathrm{R}^{k+\ell}$ be a definable set. Let $\pi_{1}: \mathrm{R}^{k+\ell} \rightarrow \mathrm{R}^{k}$ (resp. $\pi_{2}: \mathrm{R}^{k+\ell} \rightarrow \mathrm{R}^{\ell}$ ), be the projections onto the first $k$ (resp. last $\ell$ ) co-ordinates.

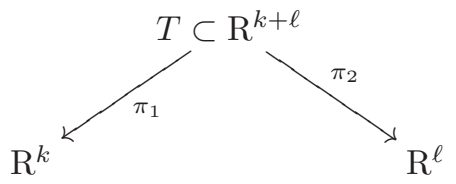

We will call a subset $S$ of $\mathrm{R}^{k}$ to be a $\left(T, \pi_{1}, \pi_{2}\right)$-set if

$$
S=\pi_{1}\left(\pi_{2}^{-1}(\mathbf{y}) \cap T\right)
$$

for some $\mathbf{y} \in \mathrm{R}^{\ell}$, and when the context is clear we will denote $T_{\mathbf{y}}=\pi_{1}\left(\pi_{2}^{-1}(\mathbf{y}) \cap T\right)$. In this paper, we will consider finite families of $\left(T, \pi_{1}, \pi_{2}\right)$-sets, where $T$ is some fixed definable set for each such family, and we will call a family of $\left(T, \pi_{1}, \pi_{2}\right)$-sets to be a $\left(T, \pi_{1}, \pi_{2}\right)$-family. We will also sometimes refer to a finite $\left(T, \pi_{1}, \pi_{2}\right)$-family as an arrangement of $\left(T, \pi_{1}, \pi_{2}\right)$-sets.

For any definable set $X \subset \mathrm{R}^{k}$, we let $b_{i}(X)$ denote the $i$-th Betti number of $X$, and we let $b(X)$ denote $\sum_{i \geq 0} b_{i}(X)$. We define the topological complexity of an arrangement $\mathcal{A}$ of $\left(T, \pi_{1}, \pi_{2}\right)$-sets to be the number

$$
\sum_{D \in \mathcal{C}(\mathcal{A})} \sum_{i=0}^{k} b_{i}(D)
$$

REMARK 1. We remark here that for o-minimal structures over an arbitrary real closed field R, ordinary singular homology is not well defined. Even though o-minimal versions of singular co-homology theory, as well Čech co-homology theory, has been developed recently (see [14, 15), in this paper we take a simpler approach and use a modified homology theory (which agrees with singular homology in case $R=\mathbb{R}$ and which is homotopy invariant) as done in [5] in case of semi-algebraic sets over arbitrary real closed fields (see [5], page 279). The underlying idea behind that definition is as follows. Since closed and bounded semi-algebraic (as well as definable) sets are finitely triangulable, simplicial homology is well defined for such sets. Furthermore, it is shown in [6 that it is possible to replace an arbitrary semi-algebraic set by a closed and bounded one which is homotopy equivalent to the original set. We prove an analogous result for arbitrary definable sets in this paper (see Theorem 3.3 below). We now define the homology groups of the original set to be the simplicial homology groups of the closed and bounded definable set which is homotopy equivalent to it. It is clear that this definition is homotopy invariant.

We now give a few examples to show that arrangements of objects of bounded description complexities are included in the class of arrangements we study, but our class is much larger since $T$ need not be semi-algebraic.

\subsubsection{Examples}

Example 4. Let $\mathcal{S}(\mathrm{R})$ be the o-minimal structure $\mathcal{S}_{\mathrm{sa}}(\mathrm{R})$. Let $T \subset \mathrm{R}^{2 k+1}$ be the semialgebraic set defined by

$$
T=\left\{\left(x_{1}, \ldots, x_{k}, a_{1}, \ldots, a_{k}, b\right) \mid\langle\mathbf{a}, \mathbf{x}\rangle-b=0\right\}
$$


(where we denote $\mathbf{a}=\left(a_{1}, \ldots, a_{k}\right)$ and $\mathbf{x}=\left(x_{1}, \ldots, x_{k}\right)$ ), and $\pi_{1}$ and $\pi_{2}$ are the projections onto the first $k$ and last $k+1$ co-ordinates respectively. A $\left(T, \pi_{1}, \pi_{2}\right)$-set is clearly a hyperplane in $\mathrm{R}^{k}$ and vice versa.

EXAmple 5. Again, let $\mathcal{S}(\mathrm{R})$ be the o-minimal structure $\mathcal{S}_{\text {sa }}(\mathrm{R})$. Let $T \subset \mathrm{R}^{k+k(k+1)}$ be the semi-algebraic set defined by

$$
T=\left\{\left(\mathbf{x}, \mathbf{y}_{0}, \ldots, \mathbf{y}_{k}\right) \mid \mathbf{x}, \mathbf{y}_{0}, \ldots, \mathbf{y}_{k} \in \mathrm{R}^{k}, \mathbf{x} \in \operatorname{conv}\left(\mathbf{y}_{0}, \ldots, \mathbf{y}_{k}\right)\right\},
$$

and $\pi_{1}$ and $\pi_{2}$ the projections onto the first $k$ and last $k(k+1)$ co-ordinates respectively. A $\left(T, \pi_{1}, \pi_{2}\right)$-set is a (possibly degenerate) $k$-simplex in $\mathrm{R}^{k}$ and vice versa.

Arrangements of hyperplanes as well as simplices have been well studied in computational geometry, and thus the two previous examples do not introduce anything new. We now discuss an example which could not be handled by the existing techniques in computational geometry, such as linearization.

Example 6 . Now, let $\mathcal{S}(\mathbb{R})$ be the o-minimal structure $\mathcal{S}_{\exp }(\mathbb{R})$. Let $T \subset \mathbb{R}^{k+m(k+1)}$ be the set defined by

$$
\begin{gathered}
T=\left\{\left(\mathbf{x}, \mathbf{y}_{1}, \ldots, \mathbf{y}_{m}, a_{1}, \ldots, a_{m}\right) \mid \mathbf{x}, \mathbf{y}_{1}, \ldots, \mathbf{y}_{m} \in \mathbb{R}^{k}, a_{1}, \ldots, a_{m} \in \mathbb{R},\right. \\
\left.x_{1}, \ldots, x_{k}>0, \sum_{i=0}^{m} a_{i} \mathbf{x}^{\mathbf{y}_{i}}=0\right\},
\end{gathered}
$$

and $\pi_{1}: \mathbb{R}^{k+m(k+1)} \rightarrow \mathbb{R}^{k}$ and $\pi_{2}: \mathbb{R}^{k+m(k+1)} \rightarrow \mathbb{R}^{m(k+1)}$ be the projections onto the first $k$ and last $m(k+1)$ co-ordinates respectively. It can be shown that $T$ is definable in the structure $\mathcal{S}_{\exp }(\mathbb{R})$. The $\left(T, \pi_{1}, \pi_{2}\right)$-sets in this example include (amongst others) all semi-algebraic sets consisting of intersections with the positive orthant of all real algebraic sets defined by a polynomial having at most $m$ monomials (different sets of monomials are allowed to occur in different polynomials).

Definition 3. Let $\mathcal{A}=\left\{S_{1}, \ldots, S_{n}\right\}$, such that each $S_{i} \subset \mathrm{R}^{k}$ is a $\left(T, \pi_{1}, \pi_{2}\right)$-set. For $I \subset$ $\{1, \ldots, n\}$, we let $\mathcal{A}(I)$ denote the set

$$
\bigcap_{i \in I \subset[1 \ldots n]} S_{i} \cap \bigcap_{j \in[1 \ldots n] \backslash I} \mathrm{R}^{k} \backslash S_{j},
$$

and we will call such a set to be a basic $\mathcal{A}$-set. We will denote by $\mathcal{C}(\mathcal{A})$ the set of non-empty connected components of all basic $\mathcal{A}$-sets.

We will call definable subsets $S \subset \mathrm{R}^{k}$ defined by a Boolean formula whose atoms are of the form, $x \in S_{i}, 1 \leq i \leq n$, a $\mathcal{A}$-set. A $\mathcal{A}$-set is thus a union of basic $\mathcal{A}$-sets. If $T$ is closed, and the Boolean formula defining $S$ has no negations, then $S$ is closed by definition (since each $S_{i}$ being homeomorphic to the intersection of $T$ with a closed set $\pi^{-1}(\mathbf{y})$ for some $\mathbf{y} \in \mathrm{R}^{\ell}$ is closed) and we call such a set an $\mathcal{A}$-closed set.

Moreover, if $V$ is any closed definable subset of $\mathrm{R}^{k}$, and $S$ is an $\mathcal{A}$-set (resp. $\mathcal{A}$-closed set), then we will call $S \cap V$ an $(\mathcal{A}, V)$-set (resp. $(\mathcal{A}, V)$-closed set).

\subsection{Known properties}

Definable families of sets in an o-minimal structure (such as those defined above) have been studied and they satisfy important finiteness properties similar to those of semi-algebraic 
families. We list here a couple of properties which are important in the combinatorial study of arrangements.

\subsubsection{Finiteness of topological types}

Theorem 1.1 30, 12. Let $\mathcal{S}(\mathrm{R})$ be an o-minimal structure over a real closed field $\mathrm{R}$ and let $T \subset \mathrm{R}^{k+\ell}$ be a closed definable set. Then, the number of homeomorphism types amongst $\left(T, \pi_{1}, \pi_{2}\right)$-sets is finite.

REMARK 2. Note that, since the sum of the Betti number of any definable set is finite (since they are finitely triangulable [12, Theorem 4.4]) Theorem 1.1] implies that there exists a constant $C=C(T)$ (depending only on $T$ ) such that for any $\left(T, \pi_{1}, \pi_{2}\right)$-set $S$,

$$
\sum_{i=0}^{k} b_{i}(S) \leq C .
$$

1.6.2. Finiteness of $V C$ dimension The notion of Vapnik-Chervonenkis dimension is important in many applications in computational geometry (see [22]). We note here that $\left(T, \pi_{1}, \pi_{2}\right)$-families have finite Vapnik-Chervonenkis dimension, for any fixed definable $T \subset$ $\mathrm{R}^{k+\ell}$. The following result is proved in $[\mathbf{3 0}$.

We first recall the definition of the Vapnik-Chervonenkis dimension.

Definition 4 [22. Let $\mathcal{F}$ be a set of subsets of an infinite set $X$. We say that a finite subset $A \subset X$ is shattered by $\mathcal{F}$ if each subset $B$ of $A$ can be expressed as $F_{B} \cap A$ for some $F_{B} \in \mathcal{F}$. The VC-dimension of $\mathcal{F}$ is defined as

$$
\sup _{A \subset X,|A|<\infty, A \text { is shattered by } \mathcal{F}}|A| .
$$

Theorem 1.2 [30]. Let $T$ be some definable subset of $\mathrm{R}^{k+\ell}$ in some o-minimal structure $\mathcal{S}(\mathrm{R})$, and $\pi_{1}: \mathrm{R}^{k+\ell} \rightarrow \mathrm{R}^{k}, \pi_{2}: \mathrm{R}^{k+\ell} \rightarrow \mathrm{R}^{\ell}$ the two projections. Then the VC-dimension of the family of $\left(T, \pi_{1}, \pi_{2}\right)$-sets is finite.

\section{Main results}

In this section we state our main results. As stated in the Introduction, our goal is to study the combinatorial and topological complexity of sets defined in terms of $n$ definable sets belonging to a fixed definable family in terms of the parameter $n$. We show that the basic results on combinatorial and topological complexity of arrangements continue to hold in this setting. Finally, as a sample application of our results we extend a recent result of Alon et al. [3] on crossing patterns of semi-algebraic sets to the o-minimal setting.

REMARK 3. As remarked earlier, in many results on bounding the combinatorial complexity of arrangements (of sets of constant description complexity) there is an assumption that the sets be in general position [1. This is a rather strong assumption and enables one to assume, for instance, if the sets of the arrangements are hypersurfaces, that they intersect transversally and this property usually plays a crucial role in the proof. In this paper we make no assumptions on general positions, nor on the objects of the arrangement themselves (apart from the fact 
that they come from a fixed definable family). The homological methods used in this paper make such assumptions unnecessary.

\subsection{Combinatorial and topological complexity of arrangements}

TheOREm 2.1. Let $\mathcal{S}(\mathrm{R})$ be an o-minimal structure over a real closed field $\mathrm{R}$ and let $T \subset \mathrm{R}^{k+\ell}$ be a closed definable set. Then, there exists a constant $C=C(T)>0$ depending only on $T$, such that for any $\left(T, \pi_{1}, \pi_{2}\right)$-family $\mathcal{A}=\left\{S_{1}, \ldots, S_{n}\right\}$ of subsets of $\mathrm{R}^{k}$ the following holds.

(i) For every $i, 0 \leq i \leq k$,

$$
\sum_{D \in \mathcal{C}(\mathcal{A})} b_{i}(D) \leq C \cdot n^{k-i}
$$

In particular, the combinatorial complexity of $\mathcal{A}$, which is equal to

$$
\sum_{D \in \mathcal{C}(\mathcal{A})} b_{0}(D)
$$

is at most $C \cdot n^{k}$.

(ii) The topological complexity of any $m$ cells in the arrangement $\mathcal{A}$ is bounded by $m+C$. $n^{k-1}$.

Since dimension is a definable invariant (see [30]) we can refine the notions of combinatorial and topological complexity to arrangements restricted to a definable set of possibly smaller dimension than that of the ambient space as follows.

Let $V$ be a closed definable subset of $\mathrm{R}^{k}$ of dimension $k^{\prime} \leq k$. For any $\left(T, \pi_{1}, \pi_{2}\right)$-family, $\mathcal{A}=\left\{S_{1}, \ldots, S_{n}\right\}$, of subsets of $\mathrm{R}^{k}$, and $I \subset\{1, \ldots, n\}$, we let $\mathcal{A}(I, V)$ denote the set

$$
V \cap \bigcap_{i \in I \subset[1 \ldots n]} S_{i} \cap \bigcap_{j \in[1 \ldots n] \backslash I} \mathrm{R}^{k} \backslash S_{j},
$$

and we call a connected component of $\mathcal{A}(I, V)$ a cell of the arrangement restricted to $V$.

Let $\mathcal{C}(\mathcal{A}, V)$ denote the set of all non-empty cells of the arrangement $\mathcal{A}$ restricted to $V$, and we call the cardinality of $\mathcal{C}(\mathcal{A}, V)$ the combinatorial complexity of the arrangement $\mathcal{A}$ restricted to $V$. Similarly, we define the topological complexity of an arrangement $\mathcal{A}$ restricted to $V$ to be the number

$$
\sum_{D \in \mathcal{C}(\mathcal{A}, V)} \sum_{i=0}^{k^{\prime}} b_{i}(D) .
$$

We have the following generalization of Theorem 2.1.

TheOrem 2.2. Let $\mathcal{S}(\mathrm{R})$ be an o-minimal structure over a real closed field $\mathrm{R}$ and let $T \subset \mathrm{R}^{k+\ell}, V \subset \mathrm{R}^{k}$ be closed definable sets with $\operatorname{dim}(V)=k^{\prime}$. Then, there exists a constant $C=$ $C(T, V)>0$ depending only on $T$ and $V$, such that for any $\left(T, \pi_{1}, \pi_{2}\right)$-family, $\mathcal{A}=\left\{S_{1}, \ldots, S_{n}\right\}$, of subsets of $\mathrm{R}^{k}$, and for every $i, 0 \leq i \leq k^{\prime}$,

$$
\sum_{D \in \mathcal{C}(\mathcal{A}, V)} b_{i}(D) \leq C \cdot n^{k^{\prime}-i}
$$

In particular, the combinatorial complexity of $\mathcal{A}$ restricted to $V$, which is equal to $\sum_{D \in \mathcal{C}(\mathcal{A}, V)} b_{0}(D)$, is bounded by $C \cdot n^{k^{\prime}}$. 
Now, let as before $\mathcal{S}(\mathrm{R})$ be an o-minimal structure over a real closed field $\mathrm{R}$, and let $T \subset \mathrm{R}^{k+\ell}$, $V \subset \mathrm{R}^{k}$ be closed definable sets with $\operatorname{dim}(V)=k^{\prime}$.

TheOREM 2.3. Let $\mathcal{S}(\mathrm{R})$ be an o-minimal structure over a real closed field $\mathrm{R}$, and let $T \subset \mathrm{R}^{k+\ell}, V \subset \mathrm{R}^{k}$ be closed definable sets with $\operatorname{dim}(V)=k^{\prime}$. Then, there exists a constant $C=C(T, V)>0$ such that for any $\left(T, \pi_{1}, \pi_{2}\right)$-family, $\mathcal{A}$ with $|\mathcal{A}|=n$, and an $\mathcal{A}$-closed set $S_{1} \subset \mathrm{R}^{k}$, and an $\mathcal{A}$-set $S_{2} \subset \mathrm{R}^{k}$,

$$
\begin{gathered}
\sum_{i=0}^{k^{\prime}} b_{i}\left(S_{1} \cap V\right) \leq C \cdot n^{k^{\prime}} \text { and, } \\
\sum_{i=0}^{k^{\prime}} b_{i}\left(S_{2} \cap V\right) \leq C \cdot n^{2 k^{\prime}} .
\end{gathered}
$$

\subsection{Topological complexity of projections}

In Theorem 2.3 we obtained bounds on the topological complexity of definable sets belonging to the Boolean algebra of sets generated by any $\left(T, \pi_{1}, \pi_{2}\right)$-family of sets of cardinality $n$. We now consider the images of such sets under linear projections. Such projections are closely related to the classical problem of quantifier elimination, and play a very important role in semi-algebraic geometry. In the case of semi-algebraic sets, there exist effective algorithms for performing quantifier elimination, which enable one to compute semi-algebraic descriptions of projections of semi-algebraic sets in an efficient manner (see, for instance, 5]). Notice however that unlike in the case of semi-algebraic sets, we do not have effective algorithms for performing quantifier elimination over a general o-minimal structure.

Using our theorem on quantitative cylindrical definable cell decomposition (Theorem 2.5] below) it is possible to give a doubly exponential bound (of the form $C(T) \cdot n^{2\left(2^{k}-1\right)}$ ) on the sum of the Betti numbers of such projections. However, adapting a spectral sequence argument from 18, we have the following singly exponential bound.

THEOREM 2.4 (Topological complexity of projections). Let $\mathcal{S}(\mathrm{R})$ be an o-minimal structure, and let $T \subset \mathrm{R}^{k+\ell}$ be a definable, closed and bounded set. Let $k=k_{1}+k_{2}$ and let $\pi_{3}: \mathrm{R}^{k} \rightarrow \mathrm{R}^{k_{2}}$ denote the projection map on the last $k_{2}$ co-ordinates. Then, there exists a constant $C=C(T)>0$ such that for any $\left(T, \pi_{1}, \pi_{2}\right)$-family, $\mathcal{A}$, with $|\mathcal{A}|=n$, and an $\mathcal{A}$-closed set $S \subset \mathrm{R}^{k}$,

$$
\sum_{i=0}^{k_{2}} b_{i}\left(\pi_{3}(S)\right) \leq C \cdot n^{\left(k_{1}+1\right) k_{2}} .
$$

\subsection{Cylindrical definable cell decompositions}

In semi-algebraic geometry, cylindrical algebraic decomposition is a very important method for obtaining a decomposition of an arbitrary semi-algebraic set into topological balls of various dimensions. Once such a decomposition is computed, it can be refined to a semi-algebraic triangulation, and various topological information about a given semi-algebraic set (such as

its Betti numbers) can be computed easily from such a triangulation. Moreover, cylindrical algebraic decomposition can also be used for solving the quantifier elimination problem (see [5] for an exposition and pointers to the large amount of literature on this subject). 
The analogue of cylindrical algebraic decomposition over an o-minimal structure is called Cylindrical Definable Cell Decomposition. We first recall the definition of Cylindrical Definable Cell Decomposition (henceforth called $c d c d$ ) following [12.

Definition 5. A cded of $\mathrm{R}^{k}$ is a finite partition of $\mathrm{R}^{k}$ into definable sets $\left(C_{i}\right)_{i \in I}$ (called the cells of the cdcd) satisfying the following properties.

(i) If $k=1$ then a cdcd of $\mathrm{R}$ is given by a finite set of points $a_{1}<\cdots<a_{N}$ and the cells of the cdcd are the singletons $\left\{a_{i}\right\}$ as well as the open intervals, $\left(\infty, a_{1}\right),\left(a_{1}, a_{2}\right), \ldots,\left(a_{N}, \infty\right)$.

(ii) If $k>1$, then a cded of $\mathrm{R}^{k}$ is given by a cdcd, $\left(C_{i}^{\prime}\right)_{i \in I^{\prime}}$, of $\mathrm{R}^{k-1}$ and for each $i \in I^{\prime}$, a collection of cells, $\mathcal{C}_{i}$ defined by

$$
\mathcal{C}_{i}=\left\{\phi_{i}\left(C_{i}^{\prime} \times D_{j}\right) \mid j \in J_{i}\right\},
$$

where

$$
\phi_{i}: C_{i}^{\prime} \times \mathrm{R} \rightarrow \mathrm{R}^{k}
$$

is a definable homeomorphism satisfying $\pi \circ \phi=\pi,\left(D_{j}\right)_{j \in J_{i}}$ is a cdcd of R, and $\pi$ : $\mathrm{R}^{k} \rightarrow \mathrm{R}^{k-1}$ is the projection map onto the first $k-1$ coordinates. The cdcd of $\mathrm{R}^{k}$ is then given by

$$
\bigcup_{i \in I^{\prime}} \mathcal{C}_{i}
$$

Given a family of definable subsets $\mathcal{A}=\left\{S_{1}, \ldots, S_{n}\right\}$ of $\mathrm{R}^{k}$, we say that a cdcd is adapted to $\mathcal{A}$, if each $S_{i}$ is a union of cells of the given cdcd.

The fact that given any finite family $\mathcal{A}$ of definable subsets of $\mathrm{R}^{k}$, there exists a cdcd of $\mathrm{R}^{k}$ adapted to $\mathcal{A}$ is classical (see [12, 30]). However, for the purposes of this paper we need a quantitative version of this result. Such quantitative versions are known in the semi-algebraic as well as semi-Pfaffian categories (see, for example, [5, 20]), but is missing in the general o-minimal setting.

Given a $\left(T, \pi_{1}, \pi_{2}\right)$-family $\mathcal{A}$ of cardinality $n$, we give a bound on the size of a cdcd of $\mathrm{R}^{k}$ adapted to this family in terms of $n$, and furthermore show that cells of the cdcd come from a definable family which depends only on $T$ (independent of $n$ ) and each such cell can be defined only in terms of a constant number of elements of $\mathcal{A}$. This latter property is essential in the combinatorial application described later in the paper.

Since we will need to consider several different projections, we adopt the following convention. Given $m$ and $p, p \leq m$, we will denote by $\pi_{m}^{\leq p}: \mathrm{R}^{m} \rightarrow \mathrm{R}^{p}\left(\right.$ resp. $\pi_{m}^{>p}: \mathrm{R}^{m} \rightarrow \mathrm{R}^{m-p}$ ) the projection onto the first $p$ (resp. the last $m-p$ ) coordinates.

We prove the following theorem.

Theorem 2.5 (Quantitative cylindrical definable cell decomposition). Let $\mathcal{S}(\mathrm{R})$ be an ominimal structure over a real closed field $\mathrm{R}$, and let $T \subset \mathrm{R}^{k+\ell}$ be a closed definable set. Then, there exist constants $C_{1}, C_{2}>0$ depending only on $T$, and definable sets,

$$
\left\{T_{\alpha}\right\}_{\alpha \in I}, T_{\alpha} \subset \mathrm{R}^{k} \times \mathrm{R}^{2\left(2^{k}-1\right) \cdot \ell},
$$

depending only on $T$, with $|I| \leq C_{1}$, such that for any $\left(T, \pi_{1}, \pi_{2}\right)$-family, $\mathcal{A}=\left\{S_{1}, \ldots, S_{n}\right\}$ with $S_{i}=T_{\mathbf{y}_{i}}, \mathbf{y}_{i} \in \mathrm{R}^{\ell}, 1 \leq i \leq n$, some sub-collection of the sets

$$
\begin{gathered}
\pi_{k+2\left(2^{k}-1\right) \cdot \ell}^{\leq k}\left(\pi_{k+2\left(2^{k}-1\right) \cdot \ell}^{>k}\left(\mathbf{y}_{i_{1}}, \ldots, \mathbf{y}_{i_{2\left(2^{k}-1\right)}}\right) \cap T_{\alpha}\right), \\
\alpha \in I, 1 \leq i_{1}, \ldots, i_{2\left(2^{k}-1\right)} \leq n,
\end{gathered}
$$


form a cdcd of $\mathrm{R}^{k}$ compatible with $\mathcal{A}$. Moreover, the cdcd has at most $C_{2} \cdot n^{2\left(2^{k}-1\right)}$ cells.

The combinatorial complexity bound in Theorem 2.5 compares favorably with the combinatorial parts of similar quantitative results on cylindrical decomposition of semi-algebraic sets (see for instance, Section 11.1 in [5]), as well as sub-Pfaffian sets (see the main result in [16]). Moreover, since a doubly exponential dependence on $k$ is unavoidable (see [13]), the complexity bound in Theorem 2.5 is very close to the best possible. Notice also that it is possible to use Theorem 2.5 to give a doubly exponential bound on the Betti numbers of an $\mathcal{A}$-closed set. However, we prove much better (singly exponential) bounds on the Betti numbers of such sets (Theorems 2.1 and 2.2) using different techniques.

\subsection{Application}

We end with an application (Theorem 2.6 below) which generalizes a Ramsey-type result due to Alon et al. 3 from the class of semi-algebraic sets of constant description complexity to $\left(T, \pi_{1}, \pi_{2}\right)$-families. One immediate consequence of Theorem 2.6 is that if we have two $\left(T, \pi_{1}, \pi_{2}\right)$-families, $\mathcal{A}$ and $\mathcal{B}$ of sufficiently large size, then one can always find a constant fraction, $\mathcal{A}^{\prime} \subset \mathcal{A}, \mathcal{B}^{\prime} \subset \mathcal{B}$ of each, having the property that either every pair $(A, B) \in \mathcal{A}^{\prime} \times \mathcal{B}^{\prime}$ satisfy some definable relation (for example, having a non-empty intersection) or no pair in $\mathcal{A}^{\prime} \times \mathcal{B}^{\prime}$ satisfy that relation.

More precisely,

TheOREm 2.6. Let $\mathcal{S}(\mathrm{R})$ be an o-minimal structure over a real closed field $\mathrm{R}$, and let $F$ be a closed definable subset of $\mathrm{R}^{\ell} \times \mathrm{R}^{\ell}$. Then, there exists a constant $1>\varepsilon=\varepsilon(F)>0$, depending only on $F$, such that for any set of $n$ points,

$$
\mathcal{F}=\left\{\mathbf{y}_{1}, \ldots, \mathbf{y}_{n} \in \mathrm{R}^{\ell}\right\}
$$

there exists two subfamilies $\mathcal{F}_{1}, \mathcal{F}_{2} \subset \mathcal{F}$, with $\left|\mathcal{F}_{1}\right|,\left|\mathcal{F}_{2}\right| \geq \varepsilon n$ and either,

(i) for all $\mathbf{y}_{i} \in \mathcal{F}_{1}$ and $\mathbf{y}_{j} \in \mathcal{F}_{2},\left(\mathbf{y}_{i}, \mathbf{y}_{j}\right) \in F$, or

(ii) for no $\mathbf{y}_{i} \in \mathcal{F}_{1}$ and $\mathbf{y}_{j} \in \mathcal{F}_{2},\left(\mathbf{y}_{i}, \mathbf{y}_{j}\right) \in F$.

An interesting application of Theorem 2.6 is the following.

Corollary 2.7. Let $\mathcal{S}(\mathrm{R})$ be an o-minimal structure over a real closed field $\mathrm{R}$, and let $T \subset \mathrm{R}^{k+\ell}$ be a closed definable set. Then, there exists a constant $1>\varepsilon=\varepsilon(T)>0$ depending only on $T$, such that for any $\left(T, \pi_{1}, \pi_{2}\right)$-family, $\mathcal{A}=\left\{S_{1}, \ldots, S_{n}\right\}$, there exists two subfamilies $\mathcal{A}_{1}, \mathcal{A}_{2} \subset \mathcal{A}$, with $\left|\mathcal{A}_{1}\right|,\left|\mathcal{A}_{2}\right| \geq \varepsilon n$, and either,

(i) for all $S_{i} \in \mathcal{A}_{1}$ and $S_{j} \in \mathcal{A}_{2}, S_{i} \cap S_{j} \neq \emptyset$ or

(ii) for all $S_{i} \in \mathcal{A}_{1}$ and $S_{j} \in \mathcal{A}_{2}, S_{i} \cap S_{j}=\emptyset$.

\section{Proofs of the main results}

We first need a few preliminary results.

\subsection{Finite unions of definable families}

Suppose that $T_{1}, \ldots, T_{m} \subset \mathrm{R}^{k+\ell}$ are closed, definable sets, $\pi_{1}: \mathrm{R}^{k+\ell} \rightarrow \mathrm{R}^{k}$ and $\pi_{2}: \mathrm{R}^{k+\ell} \rightarrow$ $\mathrm{R}^{\ell}$ the two projections. 
We show that there exists a a certain closed definable subset $T^{\prime} \subset \mathrm{R}^{k+\ell+m}$ depending only on $T_{1}, \ldots, T_{m}$, such that for any collection of $\left(T_{i}, \pi_{1}, \pi_{2}\right)$ families $\mathcal{A}_{i}, 1 \leq i \leq m$, the union, $\cup_{1 \leq i \leq m} \mathcal{A}_{i}$, is a $\left(T^{\prime}, \pi_{1}^{\prime}, \pi_{2}^{\prime}\right)$-family, where $\pi_{1}^{\prime}: \mathrm{R}^{k+m+\ell} \rightarrow \mathrm{R}^{k}$ and $\pi_{2}^{\prime}: \mathrm{R}^{k+\ell+m} \rightarrow \mathrm{R}^{\ell+m}$ are the usual projections.

Lemma 3.1. The family $\cup_{1 \leq i \leq m} \mathcal{A}_{i}$ is a $\left(T^{\prime}, \pi_{1}^{\prime}, \pi_{2}^{\prime}\right)$ family where,

$$
T^{\prime}=\bigcup_{i=1}^{m} T_{i} \times\left\{e_{i}\right\} \subset \mathrm{R}^{k+\ell+m},
$$

with $e_{i}$ the $i$-th standard basis vector in $\mathrm{R}^{m}$, and $\pi_{1}^{\prime}: \mathrm{R}^{k+\ell+m} \rightarrow \mathrm{R}^{k}$ and $\pi_{2}^{\prime}: \mathrm{R}^{k+\ell+m} \rightarrow \mathrm{R}^{\ell+m}$, the projections onto the first $k$ and the last $\ell+m$ coordinates respectively.

Proof. Obvious.

\subsection{Hardt triviality for definable sets}

Our main technical tool will be the following o-minimal version of Hardt's triviality theorem (see [30, 12]).

Let $X \subset \mathrm{R}^{k} \times \mathrm{R}^{\ell}$ and $A \subset \mathrm{R}^{\ell}$ be definable subsets of $\mathrm{R}^{k} \times \mathrm{R}^{\ell}$ and $\mathrm{R}^{\ell}$ respectively, and let $\pi: X \rightarrow \mathrm{R}^{\ell}$ denote the projection map.

We say that $X$ is definably trivial over $A$ if there exists a definable set $F$ and a definable homeomorphism

$$
h: F \times A \rightarrow X \cap \pi^{-1}(A)
$$

such that the following diagram commutes:

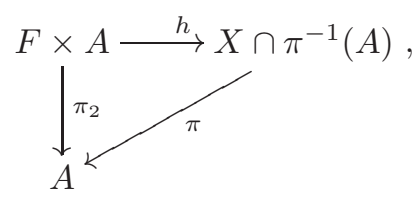

where $\pi_{2}: F \times A \rightarrow A$ is the projection onto the second factor. We call $h$ a definable trivialization of $X$ over $A$.

If $Y$ is a definable subset of $X$, we say that the trivialization $h$ is compatible with $Y$ if there is a definable subset $G$ of $F$ such that $h(G \times A)=Y \cap \pi^{-1}(A)$. Clearly, the restriction of $h$ to $G \times A$ is a trivialization of $Y$ over $A$.

TheOREM 3.2 (Hardt's theorem for definable families). Let $X \subset \mathrm{R}^{k} \times \mathrm{R}^{\ell}$ be a definable set and let $Y_{1}, \ldots, Y_{m}$ be definable subsets of $X$. Then, there exists a finite partition of $\mathrm{R}^{\ell}$ into definable sets $C_{1}, \ldots, C_{N}$ such that $X$ is definably trivial over each $C_{i}$, and moreover the trivializations over each $C_{i}$ are compatible with $Y_{1}, \ldots, Y_{m}$.

REMARK 4. Note that in particular it follows from Theorem 3.2, that there are only a finite number of topological types amongst the fibers of any definable map $f: X \rightarrow Y$ between definable sets $X$ and $Y$ (see Remark 2 ). 


\subsection{Some notation}

For any definable set $X \subset \mathrm{R}^{k}$ we will denote by $X^{c}$ the complement of $X$, and by $\bar{X}$ the closure of $X$ in $\mathrm{R}^{k}$. We also denote by $B_{k}(\mathbf{x}, r)$ (resp. $\left.\bar{B}_{k}(\mathbf{x}, r)\right)$ the open (resp. closed) ball in $\mathrm{R}^{k}$ of radius $r$ centered at $x$.

For any closed definable subset $X \subset \mathrm{R}^{k}$, we will denote by

$$
d_{X}: \mathrm{R}^{k} \rightarrow \mathrm{R}, \quad d_{X}(\mathbf{x})=\operatorname{dist}(\mathbf{x}, X) .
$$

Note that, it follows from the axioms in Definition 1 that $d_{X}$ is a definable function (that is a function whose graph is a definable set).

Given closed definable sets $X \subset V \subset \mathrm{R}^{k}$, and $\varepsilon>0$, we define the open tube of radius $\varepsilon$ around $X$ in $V$ to be the definable set

$$
\mathrm{OT}(X, V, \varepsilon)=\left\{\mathbf{x} \in V \mid d_{X}(\mathbf{x})<\varepsilon\right\} .
$$

Similarly, we define the closed tube of radius $\varepsilon$ around $X$ in $V$ to be the definable set

$$
\mathrm{CT}(X, V, \varepsilon)=\left\{\mathbf{x} \in V \mid d_{X}(\mathbf{x}) \leq \varepsilon\right\}
$$

the boundary of the closed tube,

$$
\operatorname{BT}(X, V, \varepsilon)=\left\{\mathbf{x} \in V \mid d_{X}(\mathbf{x})=\varepsilon\right\},
$$

and finally for $\varepsilon_{1}>\varepsilon_{2}>0$ we define the open annulus of radii $\varepsilon_{1}, \varepsilon_{2}$ around $X$ in $V$ to be the definable set

$$
\operatorname{Ann}\left(X, V, \varepsilon_{1}, \varepsilon_{2}\right)=\left\{\mathbf{x} \in V \mid \varepsilon_{2}<d_{X}(\mathbf{x})<\varepsilon_{1}\right\},
$$

and the closed annulus of radii $\varepsilon_{1}, \varepsilon_{2}$ around $X$ in $V$ to be the definable set

$$
\overline{\operatorname{Ann}}\left(X, V, \varepsilon_{1}, \varepsilon_{2}\right)=\left\{\mathbf{x} \in V \mid \varepsilon_{2} \leq d_{X}(\mathbf{x}) \leq \varepsilon_{1}\right\} .
$$

For brevity we will denote by $\mathrm{OT}\left(X, \mathrm{R}^{k}, \varepsilon\right)$ (resp. $\mathrm{CT}\left(X, \mathrm{R}^{k}, \varepsilon\right), \mathrm{BT}\left(X, \mathrm{R}^{k}, \varepsilon\right), \operatorname{Ann}\left(X, \mathrm{R}^{k}, \varepsilon\right)$, $\left.\overline{\operatorname{Ann}}\left(X, \mathrm{R}^{k}, \varepsilon\right)\right)$ by $\mathrm{OT}(X, \varepsilon)(\operatorname{resp} . \operatorname{CT}(X, \varepsilon), \operatorname{BT}(X, \varepsilon), \operatorname{Ann}(X, \varepsilon), \overline{\operatorname{Ann}}(X, \varepsilon))$.

\subsection{Replacing definable sets by closed and bounded ones maintaining homotopy type}

Let $\mathcal{A}=\left\{S_{1}, \ldots, S_{n}\right\}$ be a collection of closed, definable subsets of $\mathrm{R}^{k}$ and let $V \subset \mathrm{R}^{k}$ be a closed, and bounded definable set. In this section we adapt a construction due to Gabrielov and Vorobjov 21] for replacing any given $(\mathcal{A}, V)$-set by a closed bounded $\left(\mathcal{A}^{\prime}, V\right)$-set (where $\mathcal{A}^{\prime}$ is a new family of definable closely related to $\mathcal{A}$ ) such that the new set has the same homotopy type as the original one.

We denote by $\operatorname{In}(\mathcal{A}, V)$ the set,

$$
\{I \subset[1 \ldots n] \mid \mathcal{A}(I) \cap V \neq \emptyset\} .
$$

Let, $\varepsilon_{2 n} \gg \varepsilon_{2 n-1} \gg \cdots \gg \varepsilon_{2} \gg \varepsilon_{1}>0$ be sufficiently small.

For each $m, 0 \leq m \leq n$, we denote by $\operatorname{In}_{m}(\mathcal{A}, V)$ the set $\{I \in \operatorname{In}(\mathcal{A}, V)|| I \mid=m\}$.

Given $I \in \operatorname{In}_{m}(\mathcal{A}, V)$ denote by $\mathcal{A}(I)^{c l}$ to be the intersection of $V$ with the closed definable set

$$
\bigcap_{i \in I} \mathrm{CT}\left(S_{i}, \varepsilon_{2 m}\right) \cap \bigcap_{i \in[1 \ldots n] \backslash I} \overline{S_{i}^{c}} .
$$

and denote by $\mathcal{A}(I)^{\circ}$ the intersection of $V$ with the open definable set

$$
\bigcap_{i \in I} \mathrm{OT}\left(S_{i}, \varepsilon_{2 m-1}\right) \cap \bigcap_{i \in[1 \ldots n] \backslash I} S_{i}^{c}
$$

Notice that,

$$
\mathcal{A}(I) \subset \mathcal{A}(I)^{c l} \text {, as well as }
$$




$$
\mathcal{A}(I) \subset \mathcal{A}(I)^{\circ} .
$$

Let $X \subset V$ be a $(\mathcal{A}, V)$-set such that $X=\bigcup_{I \in \Sigma} \mathcal{A}(I) \cap V$ with $\Sigma \subset \operatorname{In}(\mathcal{A}, V)$. We denote $\Sigma_{m}=$ $\Sigma \cap \operatorname{In}_{m}(\mathcal{A}, V)$ and define a sequence of sets, $X^{m} \subset \mathrm{R}^{k}, 0 \leq m \leq n$ inductively as follows.

- Let $X^{0}=X$.

- For $0 \leq m \leq n$, we define

$$
X^{m+1}=\left(X^{m} \cup \bigcup_{I \in \Sigma_{m}} \mathcal{A}(I)^{c l}\right) \backslash \bigcup_{I \in \operatorname{In}_{m}(\mathcal{A}, V) \backslash \Sigma_{m}} \mathcal{A}(I)^{o}
$$

We denote by $X^{\prime}$ the set $X^{n+1}$.

The following theorem is similar to Theorem 8.1 in [6. All the steps in the proof of Theorem 8.1 in [6] also remain valid in the o-minimal context. One needs to replace the references to Hardt's theorem for semi-algebraic mappings by its o-minimal counterpart. Since repeating the entire proof with this minor modification would be tedious, we omit it from this paper.

Theorem 3.3. The sets $X$ and $X^{\prime}$ are definably homotopy equivalent.

REMARK 5. Very recently, after this paper was written, Gabrielov and Vorobjov [17] have given a much simpler construction for replacing an arbitrary definable set $X$ by a closed and bounded one, and if we use this new construction instead of the one described above, we obtain a slightly improved bound in Theorem 2.3 (namely $C \cdot n^{k^{\prime}}$ instead of $C \cdot n^{2 k^{\prime}}$ ).

Remark 6. Note that $X^{\prime}$ is a $\left(\mathcal{A}^{\prime}, V\right)$-closed set, where

$$
\mathcal{A}^{\prime}=\bigcup_{i, j=1}^{n}\left\{S_{i}, \mathrm{CT}\left(S_{i}, \varepsilon_{2 j}\right), \mathrm{OT}\left(S_{i}, \varepsilon_{2 j-1}\right)^{c}\right\}
$$

If $\mathcal{A}$ is a $\left(T, \pi_{1}, \pi_{2}\right)$-family for some definable closed subset $T \subset \mathrm{R}^{k+\ell}$, then by Lemma 3.1 $\mathcal{A}^{\prime}$ is a $\left(T^{\prime}, \pi_{1}^{\prime}, \pi_{2}^{\prime}\right)$-family for some definable $T^{\prime}$ depending only on $T$.

\subsection{Mayer-Vietoris inequalities}

We will need a couple of inequalities which follows from the exactness of Mayer-Vietoris sequence.

REMARK 7. Note that for a closed and bounded definable set $X \subset \mathrm{R}^{k}$, the homology groups $\mathrm{H}_{*}(X)$ are isomorphic to the simplicial homology groups of any definable triangulation of $X$ and in this case the proof of the exactness of the Mayer-Vietoris sequence is purely combinatorial in nature and presents no difficulties (even in the case when $\mathrm{R}$ is an arbitrary real closed field not necessarily equal to $\mathbb{R}$ ). The same remark also applies to arbitrary definable closed sets (not necessarily bounded), after intersecting the given sets with a large enough closed ball and using the conical structure at infinity of definable sets.

We first consider the case of two closed definable sets and then generalize to the case of many such sets.

Proposition 3.4. Let $S_{1}, S_{2}$ be two closed definable sets. Then, 


$$
\begin{gathered}
b_{i}\left(S_{1}\right)+b_{i}\left(S_{2}\right) \leq b_{i}\left(S_{1} \cup S_{2}\right)+b_{i}\left(S_{1} \cap S_{2}\right), \\
b_{i}\left(S_{1} \cup S_{2}\right) \leq b_{i}\left(S_{1}\right)+b_{i}\left(S_{2}\right)+b_{i-1}\left(S_{1} \cap S_{2}\right), \\
b_{i}\left(S_{1} \cap S_{2}\right) \leq b_{i}\left(S_{1}\right)+b_{i}\left(S_{2}\right)+b_{i+1}\left(S_{1} \cup S_{2}\right) .
\end{gathered}
$$

Let $S_{1}, \ldots, S_{n} \subset \mathrm{R}^{k}$ be closed definable sets, contained in a closed bounded definable set $V$ of dimension $k^{\prime}$. For $1 \leq t \leq n$, we let

$$
S_{\leq t}=\bigcap_{1 \leq j \leq t} S_{j}, \text { and } S^{\leq t}=\bigcup_{1 \leq j \leq t} S_{j} .
$$

Also, for $J \subset\{1, \ldots, n\}, J \neq \emptyset$, let

$$
S_{J}=\bigcap_{j \in J} S_{j} \text {, and } S^{J}=\bigcup_{j \in J} S_{j} .
$$

Finally, let $S^{\emptyset}=V$.

We have the following proposition.

Proposition 3.5. (a) For $0 \leq i \leq k^{\prime}$,

$$
b_{i}\left(S^{\leq n}\right) \leq \sum_{j=1}^{i+1} \sum_{J \subset\{1, \ldots n\}, \#(J)=j} b_{i-j+1}\left(S_{J}\right) .
$$

(b) For $0 \leq i \leq k^{\prime}$,

$$
b_{i}\left(S_{\leq n}\right) \leq b_{k^{\prime}}\left(S^{\emptyset}\right)+\sum_{j=1}^{k^{\prime}-i} \sum_{J \subset\{1, \ldots, n\}, \#(J)=j}\left(b_{i+j-1}\left(S^{J}\right)+b_{k^{\prime}}\left(S^{\emptyset}\right)\right) .
$$

Proof. See [9.

\subsection{Proof of Theorem 2.2}

We will use the following proposition in the proof of Theorem 2.2 .

Proposition 3.6. Let $\mathcal{A}=\left\{S_{1}, \ldots, S_{n}\right\}$ be a collection of closed definable subsets of $\mathrm{R}^{k}$ and let $V \subset \mathrm{R}^{k}$ be a closed, and bounded definable set. Then for all sufficiently small $1 \gg$ $\varepsilon_{1} \gg \varepsilon_{2}>0$ the following holds. For any connected component, $C$, of $\mathcal{A}(I) \cap V, I \subset[1 \ldots n]$, there exists a connected component, $D$, of the definable set

$$
\bigcap_{1 \leq i \leq n} \operatorname{Ann}\left(S_{i}, \varepsilon_{1}, \varepsilon_{2}\right)^{c} \cap V
$$

such that $D$ is definably homotopy equivalent to $C$.

Proof. The proposition will follow from the following two observations which are consequences of Theorem 3.2 (Hardt's theorem for o-minimal structures).

Observation 1. It follows from Theorem 3.2 that for all sufficiently small $\varepsilon_{1}>0$ and for each connected component $C$ of $\mathcal{A}(I) \cap V$, there exists a connected component $D^{\prime}$ of

$$
\bigcap_{i \in I} S_{i} \cap \bigcap_{j \in[1 \ldots n] \backslash I} \mathrm{OT}\left(S_{j}, \varepsilon_{1}\right)^{c} \cap V,
$$


definably homotopy equivalent to $C$.

Observation 2. For all sufficiently small, $\varepsilon_{2}$ with $0<\varepsilon_{2} \ll \varepsilon_{1}$, and for each connected component $D^{\prime}$ of

$$
\bigcap_{i \in I} S_{i} \cap \bigcap_{j \in[1 \ldots n] \backslash I} \mathrm{OT}\left(S_{j}, \varepsilon_{1}\right)^{c} \cap V
$$

there exists a connected component $D$ of

$$
W:=\bigcap_{i \in I} \mathrm{CT}\left(S_{i}, \varepsilon_{2}\right) \cap \bigcap_{j \in[1 \ldots n] \backslash I} \mathrm{OT}\left(S_{j}, \varepsilon_{1}\right)^{c} \cap V,
$$

definably homotopy equivalent to $D^{\prime}$.

Now notice that $D$ is connected and contained in the set

$$
\bigcap_{1 \leq i \leq n} \operatorname{Ann}\left(S_{i}, \varepsilon_{1}, \varepsilon_{2}\right)^{c} \cap V
$$

Let $D^{\prime \prime}$ be the connected component of

$$
\bigcap_{1 \leq i \leq n} \operatorname{Ann}\left(S_{i}, \varepsilon_{1}, \varepsilon_{2}\right)^{c} \cap V
$$

containing $D$. We claim that $D=D^{\prime \prime}$, which will prove the proposition.

Suppose $D^{\prime \prime} \backslash D \neq \emptyset$. Then, $D^{\prime \prime} \backslash W \neq \emptyset$, since otherwise $D^{\prime \prime} \subset W$, which would imply that $D^{\prime \prime}=D$, since $D^{\prime \prime}$ is connected and $D \subset D^{\prime \prime}$ is a connected component of $W$. Let $\mathbf{x} \in D^{\prime \prime} \backslash W$ and $\mathbf{y}$ any point in $D$. Since $\mathbf{x} \notin W$, either

(i) there exists $i \in I$ such that $\mathbf{x} \in \mathrm{OT}\left(S_{i}, \varepsilon_{1}\right)^{c}$ or

(ii) there exists $i \in[1 \ldots n] \backslash I$ such that $\mathrm{x} \in \mathrm{CT}\left(S_{i}, \varepsilon_{2}\right)$.

Let $\gamma:[0,1] \rightarrow D^{\prime \prime}$ be a definable path with $\gamma(0)=\mathbf{x}, \gamma(1)=\mathbf{y}$. and let $d_{i}: D^{\prime \prime} \rightarrow \mathrm{R}$ be the definable continuous function, $d_{i}(\mathbf{z})=\operatorname{dist}\left(\mathbf{z}, S_{i}\right)$.

Then, in the first case, $d_{i}(\mathbf{x})=d_{i}(\gamma(0)) \geq \varepsilon_{1}$ and $d_{i}(\mathbf{y})=d_{i}(\gamma(1))<\varepsilon_{2}$, implying that there exists $t \in(0,1)$ with $\varepsilon_{2}<d_{i}(\gamma(t))<\varepsilon_{1}$ implying that $d_{i}(\gamma(t)) \notin \operatorname{Ann}\left(S_{i}, \varepsilon_{1}, \varepsilon_{2}\right)^{c}$ and hence not in $D^{\prime \prime}$ (a contradiction). In the second case, $d_{i}(\mathbf{x})=d_{i}(\gamma(0))<\varepsilon_{2}$ and $d_{i}(\mathbf{y})=d_{i}(\gamma(1)) \geq \varepsilon_{1}$, implying that there exists $t \in(0,1)$ with $\varepsilon_{2}<d_{i}(\gamma(t))<\varepsilon_{1}$ again implying that $d_{i}(\gamma(t)) \notin$ $\operatorname{Ann}\left(S_{i}, \varepsilon_{1}, \varepsilon_{2}\right)^{c}$ and hence not in $D^{\prime \prime}$ (a contradiction).

We are now in a position to prove Theorem 2.2

Proof of Theorem 2.2. For $1 \leq i \leq n$, let $\mathbf{y}_{i} \in \mathrm{R}^{\ell}$ such that

$$
S_{i}=T_{\mathbf{y}_{i}},
$$

and let

$$
A_{i}\left(\varepsilon_{1}, \varepsilon_{2}\right)=\operatorname{Ann}\left(S_{i}, \varepsilon_{1}, \varepsilon_{2}\right)^{c} \cap V .
$$

Applying Proposition 3.5 we have for $0 \leq i \leq k^{\prime}$,

$$
b_{i}\left(\bigcap_{j=1}^{n} A_{j}\left(\varepsilon_{1}, \varepsilon_{2}\right)\right) \leq b_{k^{\prime}}(V)+\sum_{j=1}^{k^{\prime}-i} \sum_{J \subset\{1, \ldots, n\}, \#(J)=j}\left(b_{i+j-1}\left(A^{J}\left(\varepsilon_{1}, \varepsilon_{2}\right)\right)+b_{k^{\prime}}(V)\right),
$$

where $A^{J}\left(\varepsilon_{1}, \varepsilon_{2}\right)=\cup_{j \in J} A_{j}\left(\varepsilon_{1}, \varepsilon_{2}\right)$.

Notice that each $\operatorname{Ann}\left(S_{i}, \varepsilon_{1}, \varepsilon_{2}\right)^{c}, 1 \leq i \leq n$, is a $\left(\operatorname{Ann}\left(T, \varepsilon_{1}, \varepsilon_{2}\right)^{c}, \pi_{1}, \pi_{2}\right)$-set and moreover,

$$
\operatorname{Ann}\left(S_{i}, \varepsilon_{1}, \varepsilon_{2}\right)^{c}=\pi_{1}\left(\pi_{2}^{-1}\left(\mathbf{y}_{i}\right) \cap \operatorname{Ann}\left(T, \varepsilon_{1}, \varepsilon_{2}\right)^{c}\right) ; 1 \leq i \leq n .
$$

For $J \subset[1 \ldots n]$ with $|J| \leq k^{\prime}$, we will denote

$$
S^{J}\left(\varepsilon_{1}, \varepsilon_{2}\right)=\bigcup_{j \in J} \operatorname{Ann}\left(S_{j}, \varepsilon_{1}, \varepsilon_{2}\right)^{c} .
$$


Consider the definable set

$$
B_{J}\left(\varepsilon_{1}, \varepsilon_{2}\right)=\prod_{j \in J} \operatorname{Ann}\left(T, \varepsilon_{1}, \varepsilon_{2}\right) \cap \Delta,
$$

where $\Delta \subset \mathrm{R}^{|J|(k+\ell)}$ is the definable (in fact, semi-algebraic) set defined by

$$
\Delta=\left\{\left(\mathbf{x}, \mathbf{z}_{1}, \mathbf{x}, \mathbf{z}_{2}, \ldots, \mathbf{x}, \mathbf{z}_{|J|}\right) \mid \mathbf{x} \in \mathrm{R}^{k}, \mathbf{z}_{1}, \ldots, \mathbf{z}_{|J|} \in \mathrm{R}^{\ell}\right\} .
$$

The projection map $\pi_{2}$ induces a projection map,

$$
\prod_{j \in J} \pi_{2}: \mathrm{R}^{|J|(k+\ell)} \rightarrow \prod_{j \in J} \mathrm{R}^{\ell}
$$

We also have the natural projection

$$
\pi_{1}: B_{J}\left(\varepsilon_{1}, \varepsilon_{2}\right) \rightarrow \mathrm{R}^{k} .
$$

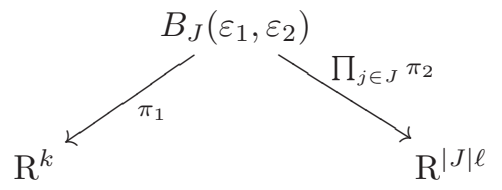

It is now easy to see that for each, $J=\left\{i_{1}, \ldots, i_{|J|}\right\}, S^{J}\left(\varepsilon_{1}, \varepsilon_{2}\right)^{c}$ is homeomorphic to $\left(\prod_{j \in J} \pi_{2}\right)^{-1}\left(\mathbf{y}_{i_{1}}, \ldots, \mathbf{y}_{i_{|J|}}\right) \cap B_{J}\left(\varepsilon_{1}, \varepsilon_{2}\right)$ via the projection $\pi_{1}$.

Using Remark 4 we can conclude there exists an upper bound depending only on $T$ (and independent of $\mathbf{y}_{1}, \ldots, \mathbf{y}_{n}$ as well as $\left.\varepsilon_{1}, \varepsilon_{2}\right)$, on the number of of topological types amongst the pairs

$$
\left(\mathrm{R}^{k}, \pi_{1}\left(\left(\prod_{j \in J} \pi_{2}\right)^{-1}\left(\mathbf{y}_{i_{1}}, \ldots, \mathbf{y}_{i_{|J|}}\right) \cap B_{J}\left(\varepsilon_{1}, \varepsilon_{2}\right)\right)\right),
$$

and hence amongst the pairs $\left(\mathrm{R}^{k}, S^{J}\left(\varepsilon_{1}, \varepsilon_{2}\right)^{c}\right)$ as well. This implies that there are only a finite number (depending on $T$ ) of topological types amongst $S^{J}\left(\varepsilon_{1}, \varepsilon_{2}\right)$. Restricting all the sets to $V$ in the above argument, we obtain that there are only finitely many (depending on $T$ and $V$ ) of topological types amongst the sets $A^{J}\left(\varepsilon_{1}, \varepsilon_{2}\right)=S^{J}\left(\varepsilon_{1}, \varepsilon_{2}\right) \cap V$.

Thus, there exists a constant $C(T, V)$ such that

$$
C(T, V)=\max _{J \subset\{1, \ldots, n\},|J| \leq k^{\prime}, 0 \leq i+j \leq k^{\prime}}\left(b_{i+j-1}\left(A^{J}\left(\varepsilon_{1}, \varepsilon_{2}\right)\right)+b_{k^{\prime}}(V)\right)+b_{k^{\prime}}(V) .
$$

It now follows from inequality 3.6 and Proposition 3.6 that,

$$
\sum_{D \in \mathcal{C}(\mathcal{A}, V)} b_{i}(D) \leq C \cdot n^{k^{\prime}-i}
$$

We now prove Theorem 2.3 .

The proof of Theorem 2.3 will follow from the following proposition. For the sake of greater clarity, and since it does not affect in any way the proof of Theorem 2.3 , we choose to be slightly less precise in the next proposition, and prove a bound on the sum of the Betti numbers of $S$ (rather than prove separate bounds on each individual Betti number). Recall from before that for any definable set $X \subset \mathrm{R}^{k}$, we denote by $b(X)$ the sum $\sum_{i \geq 0} b_{i}(X)$.

Proposition 3.7. Let $\mathcal{A}=\left\{S_{1}, \ldots, S_{n}\right\}$ be a collection of closed definable subsets of $\mathrm{R}^{k}$ and let $V \subset \mathrm{R}^{k}$ be a closed, and bounded definable set and let $S$ be an $(\mathcal{A}, V)$-closed set. Then, 
for all sufficiently small $1 \gg \varepsilon_{1} \gg \varepsilon_{2} \cdots \gg \varepsilon_{n}>0$,

$$
b(S) \leq \sum_{D \in \mathcal{C}(\mathcal{B}, V)} b(D),
$$

where

$$
\mathcal{B}=\bigcup_{i=1}^{n}\left\{S_{i}, \operatorname{BT}\left(S_{i}, \varepsilon_{i}\right), \mathrm{OT}\left(S_{i}, 2 \varepsilon_{i}\right)^{c}\right\}
$$

Proof of Proposition 3.7 We define $\mathcal{A}_{>i}=\left\{S_{i+1}, \ldots, S_{n}\right\}$ and

$$
\mathcal{B}_{i}=\left\{S_{i}, \mathrm{BT}\left(S_{i}, \varepsilon_{i}\right), \mathrm{OT}\left(S_{i}, 2 \varepsilon_{i}\right)^{c}\right\},
$$

and

$$
\mathcal{B}_{\leq i}=\left\{X \mid X=\bigcap_{j=1, \ldots, i} X_{j}, X_{j} \in \mathcal{B}_{j}\right\} .
$$

The proof of the proposition will follow from the following proposition.

Proposition 3.8. For every $(\mathcal{A}, V)$-closed set $S$,

$$
b(S) \leq \sum_{X \in \mathcal{B}_{\leq s}, X \cap V \subset S} b(X \cap V) .
$$

The main ingredient of the proof of the proposition is the following lemma.

Lemma 3.9. For every $(\mathcal{A}, V)$-closed set $S$, and every $X \in \mathcal{B}_{\leq i}$,

$$
b(S \cap X) \leq \sum_{Y \in \mathcal{B}_{i+1}} b(S \cap X \cap Y) .
$$

Proof of Lemma 3.9. Consider the sets

$$
\begin{aligned}
& T_{1}=S \cap X \cap \mathrm{OT}\left(S_{i+1}, \varepsilon_{i+1}\right)^{c}, \\
& T_{2}=S \cap X \cap \mathrm{CT}\left(S_{i+1}, 3 \varepsilon_{i+1}\right) .
\end{aligned}
$$

Clearly, $S \cap X=T_{1} \cup T_{2}$.

Using Proposition 3.4 we have that,

$$
b(S \cap X) \leq b\left(T_{1}\right)+b\left(T_{2}\right)+b\left(T_{1} \cap T_{2}\right) .
$$

Now, since

$$
\left.T_{1} \cap T_{2}=S \cap X \cap \overline{\operatorname{Ann}}\left(S_{i+1}, 3 \varepsilon_{i+1}, \varepsilon_{i+1}\right)\right),
$$

we have that,

$$
b\left(T_{1} \cap T_{2}\right)=b\left(S \cap X \cap \overline{\operatorname{Ann}}\left(S_{i+1}, 3 \varepsilon_{i+1}, \varepsilon_{i+1}\right)\right) .
$$

It is now easy to verify using Theorem 3.2 that,

$$
\begin{gathered}
T_{1} \sim S \cap X \cap \mathrm{OT}\left(S_{i+1}, 2 \varepsilon_{i+1}\right)^{c}, \\
T_{2} \sim S \cap X \cap S_{i+1}, \\
T_{1} \cap T_{2} \sim S \cap X \cap \operatorname{BT}\left(S_{i+1}, 2 \varepsilon_{i+1}\right),
\end{gathered}
$$

where $\sim$ denotes definable homotopy equivalence. 
Finally,

$$
b(S \cap X) \leq \sum_{Y \in \mathcal{B}_{i+1}} b(S \cap X \cap Y) .
$$

Proof of Proposition 3.8. Starting from the set $S$ apply Lemma 3.9 with $X$ the empty set. Now, repeatedly apply Lemma 3.9 to the terms appearing on the right-hand side of the inequality obtained, noting that for any $Y \in \mathcal{B}_{\leq s}$, either $S \cap X=X$, and thus $X \subset S$, or $S \cap X=\emptyset$.

The proof of Proposition 3.7 now follows from Proposition 3.8 .

\subsection{Proof of Theorem 2.3} 3.7

Proof of Theorem 2.3. Follows directly from Theorem 3.3 Theorem 2.2 and Proposition

\subsection{Proof of Theorem 2.4}

The proof of Theorem 2.4 relies on the bounds in Theorem 2.1 and on the following theorem which is adapted to the o-minimal setting from $\mathbf{1 8}$.

Theorem 3.10. Let $X$ and $Y$ be two closed, definable sets and $f: X \rightarrow Y$ a definable continuous surjection which is closed (i.e. $f$ takes closed sets to closed sets). Then for any integer $q$, we have

$$
b_{q}(Y) \leq \sum_{i+j=q} b_{j}\left(W_{f}^{i}(X)\right)
$$

where $W_{f}^{i}(X)$ denotes the $(i+1)$-fold fibered product of $X$ over $f$ :

$$
W_{f}^{i}(X)=\left\{\left(\mathbf{x}_{0}, \ldots, \mathbf{x}_{i}\right) \in X^{i+1} \mid f\left(\mathbf{x}_{0}\right)=\cdots=f\left(\mathbf{x}_{i}\right)\right\} .
$$

REMARK 8. (Regarding the proof of Theorem 3.10) Theorem 3.10 was proved in 18 in the semi-algebraic and semi-Pfaffian setting and follows from the existence of a spectral sequence $E_{r}^{i, j}$ converging to $H^{*}(Y)$ and such that $E_{1}^{i, j} \cong H^{j}\left(W_{f}^{i}(X)\right)$. Thus, the extension of this theorem to general o-minimal structures over arbitrary real closed fields $\mathrm{R}$ (not necessarily equal to $\mathbb{R}$ ) requires some remarks. The existence of the spectral sequence $E_{r}^{i, j}$ is a consequence of the $p$-connectivity of the $(p+1)$-fold join of any simplicial complex $K$, and the VietorisBegle theorem. The proof of the $p$-connectivity of the $(p+1)$-fold join of any simplicial complex $K$ is combinatorial in nature (see, for instance, [23, Proposition 4.4.3]), and thus presents no additional difficulties over general o-minimal structures. A purely combinatorial proof of the Vietoris-Begle theorem is also known [10, Theorem 2] (see also [17, Corollary 2.6]). Since the rest of the argument is combinatorial in nature, it extends without difficulty to closed maps in arbitrary o-minimal structures after choosing appropriate triangulations. Finally, since in any spectral sequence, the dimensions of the terms $E_{r}^{i, j}$ are non-increasing when $i$ and $j$ are fixed and $r$ increases, we obtain:

$$
b_{n}(Y)=\sum_{i+j=n} \operatorname{dim}\left(E_{\infty}^{i, j}\right) \leq \sum_{i+j=n} \operatorname{dim}\left(E_{1}^{i, j}\right),
$$

yielding inequality (3.7). 
Proof of Theorem 2.4 Notice that for each $p, 0 \leq p \leq k_{2}$, and any $\mathcal{A}$-closed set $S \subset \mathrm{R}^{k_{1}+k_{2}}$, $W_{\pi_{3}}^{p}(S) \subset \mathrm{R}^{(p+1) k_{1}+k_{2}}$ is an $\mathcal{A}^{p}$-closed set where,

$$
\begin{aligned}
\mathcal{A}^{p} & =\bigcup_{j=0}^{p} \mathcal{A}^{p, j}, \\
\mathcal{A}^{p, j} & =\bigcup_{i=1}^{n}\left\{S_{i}^{p, j}\right\},
\end{aligned}
$$

where $S_{i}^{p, j} \subset \mathrm{R}^{(p+1) k_{1}+k_{2}}$ is defined by

$$
S_{i}^{p, j}=\left\{\left(\mathbf{x}_{0}, \ldots, \mathbf{x}_{p}, \mathbf{y}\right) \mid \mathbf{x}_{j} \in \mathrm{R}^{k_{1}}, \mathbf{y} \in \mathrm{R}^{k_{2}},\left(\mathbf{x}_{j}, \mathbf{y}\right) \in S_{i}\right\} .
$$

Also, note that $\mathcal{A}^{p, j}$ is a $\left(T^{p, j}, \pi_{1}^{p}, \pi_{2}^{p}\right)$ family, where

$$
\begin{gathered}
T^{p, j}=\left\{\left(\mathbf{x}_{0}, \ldots, \mathbf{x}_{p}, \mathbf{y}, \mathbf{z}\right) \mid \mathbf{x}_{j} \in \mathrm{R}^{k_{1}}, \mathbf{y} \in \mathrm{R}^{k_{2}}, \mathbf{z} \in \mathrm{R}^{\ell},\left(\mathbf{x}_{j}, \mathbf{y}, \mathbf{z}\right) \in T\right. \\
\text { for some } j, 0 \leq j \leq p\} .
\end{gathered}
$$

and $\pi_{1}^{p}: \mathrm{R}^{(p+1) k_{1}+k_{2}+\ell} \rightarrow \mathrm{R}^{(p+1) k_{1}+k_{2}}$, and $\pi_{2}^{p}: \mathrm{R}^{(p+1) k_{1}+k_{2}+\ell} \rightarrow \mathrm{R}^{\ell}$ are the appropriate projections. Since each $T^{p, j}$ is determined by $T$, we have using Lemma 3.1 that $\mathcal{A}^{p}$ is a $\left(T^{\prime}, \pi_{1}^{\prime}, \pi_{2}^{\prime}\right)$-family for some definable $T^{\prime}$ determined by $T$. Note that, $W_{\pi_{3}}^{p}(S) \subset \mathrm{R}^{(p+1) k_{1}+k_{2}}$ is a $\mathcal{A}^{p}$-closed set and $\# \mathcal{A}^{p}=(p+1) n$. Applying Theorem 2.1 we get, for each $p$ and $j$, $0 \leq p, j<k_{2}$,

$$
b_{j}\left(W_{\pi_{3}}^{p}(S)\right) \leq C_{1}(T) \cdot n^{(p+1) k_{1}+k_{2}}
$$

The theorem now follows from Theorem 3.10 since for each $q, 0 \leq q<k_{2}$,

$$
b_{q}\left(\pi_{3}(S)\right) \leq \sum_{i+j=q} b_{j}\left(W_{\pi_{3}}^{i}(S)\right) \leq C_{2}(T) \cdot n^{(q+1) k_{1}+k_{2}} \leq C(T) \cdot n^{\left(k_{1}+1\right) k_{2}} .
$$

\subsection{Proof of Theorem 2.5}

The proof of Theorem 2.5 will follow from the following lemma (which corresponds to the first projection step in the more familiar cylindrical algebraic decomposition algorithm for semi-algebraic sets (see, for instance, [5])).

LEMma 3.11. Let $\mathcal{S}(\mathrm{R})$ be an o-minimal structure over a real closed field $\mathrm{R}$, and let $T \subset$ $\mathrm{R}^{k+\ell}$ be a closed definable set. Then, there exists definable sets $T_{1}, \ldots, T_{N} \subset \mathrm{R}^{k-1+2 \ell}$ satisfying the following. For each $i, 1 \leq i \leq N$, and $\mathbf{y}, \mathbf{y}^{\prime} \in \mathrm{R}^{\ell}$, let

$$
B_{i}\left(\mathbf{y}, \mathbf{y}^{\prime}\right)=\pi_{k-1+2 \ell}^{\leq k-1}\left(\pi_{k-1+2 \ell}^{>k-1}\left(\mathbf{y}_{1}, \mathbf{y}_{2}\right) \cap T_{i}\right) .
$$

The projection $\pi_{k}^{>1}: \mathrm{R}^{k} \rightarrow \mathrm{R}^{k-1}$ restricted to the sets $T_{\mathbf{y}} \cup T_{\mathbf{y}^{\prime}}$ is definably trivial over $B_{i}\left(\mathbf{y}, \mathbf{y}^{\prime}\right)$ and the trivialization is compatible with $T_{\mathbf{y}}$ and $T_{\mathbf{y}^{\prime}}$.

Proof. Let

$$
\begin{gathered}
V_{0}=\left\{\left(\mathbf{x}, \mathbf{y}, \mathbf{y}^{\prime}\right) \mid(\mathbf{x}, \mathbf{y}) \in T \text { or }\left(\mathbf{x}, \mathbf{y}^{\prime}\right) \in T\right\}, \\
V_{1}=\left\{\left(\mathbf{x}, \mathbf{y}, \mathbf{y}^{\prime}\right) \mid(\mathbf{x}, \mathbf{y}) \in T\right\}, \\
V_{2}=\left\{\left(\mathbf{x}, \mathbf{y}, \mathbf{y}^{\prime}\right) \mid\left(\mathbf{x}, \mathbf{y}^{\prime}\right) \in T\right\} .
\end{gathered}
$$

Note that $V_{0} \subset \mathrm{R}^{k+2 \ell}$ and $V_{1}, V_{2} \subset V_{0}$ and $V_{0}, V_{1}, V_{2}$ are all definable and determined by $T$. Applying Hardt's triviality theorem to the sets $V_{0}, V_{1}, V_{2}$ and the projection map $\pi_{k+2 \ell}^{>1}$, we 
get a definable partition of $\mathrm{R}^{k-1+2 \ell}$ into definable sets $T_{1}, \ldots, T_{N}$, such that $\left.\pi_{k+2 \ell}^{>1}\right|_{V_{0}}$ can be trivialized over each $T_{i}$ and the trivializations respects the subsets $V_{1}, V_{2}$. It is now easy to check that the sets $T_{i}$ have the required properties.

Proof of Theorem 2.5. We will use induction on $k$.

The base case is when $k=1$ and the theorem is clearly true in this case.

Now suppose by induction hypothesis that the theorem is true for $k-1$. We first apply Lemma 3.11 to obtain definable sets $T_{1}, \ldots, T_{N} \subset \mathrm{R}^{k-1+2 \ell}$ satisfying the following conditions.

For each $i, 1 \leq i \leq N$, and $\mathbf{y}, \mathbf{y}^{\prime} \in \mathrm{R}^{\ell}$, the projection $\pi_{k}^{>1}: \mathrm{R}^{k} \rightarrow \mathrm{R}^{k-1}$ restricted to the sets $T_{\mathbf{y}} \cup T_{\mathbf{y}^{\prime}}$ is definably trivial over $B_{i}\left(\mathbf{y}, \mathbf{y}^{\prime}\right)$ and the trivialization is compatible with $T_{\mathbf{y}}$ and $T_{\mathbf{y}^{\prime}}$, where

$$
B_{i}\left(\mathbf{y}, \mathbf{y}^{\prime}\right)=\pi_{k-1+2 \ell}^{\leq k-1}\left(\pi_{k-1+2 \ell}^{>k-1}-1\left(\mathbf{y}, \mathbf{y}^{\prime}\right) \cap T_{i}\right)
$$

Now let $T^{\prime}=\cup_{1 \leq i \leq N} B_{i} \times\left\{e_{i}\right\}$ where $e_{i}$ is that $i$-th standard basis vector in $\mathrm{R}^{N}$. Note that $T^{\prime} \subset \mathrm{R}^{k-1+2 \ell+N}$.

Applying the induction hypothesis to the triple

$$
\left(T^{\prime} \subset \mathrm{R}^{k-1+2\left(2^{k-1}-1\right) \cdot(2 \ell+N)}, \pi_{k-1+2\left(2^{k-1}-1\right) \cdot(2 \ell+N)}^{\leq k-1}, \pi_{k-1+2\left(2^{k-1}-1\right) \cdot(2 \ell+N)}^{>k-1}\right)
$$

we obtain definable sets,

$$
\left\{T_{j}^{\prime}\right\}_{j \in J}, \quad T_{j}^{\prime} \subset \mathrm{R}^{k-1} \times \mathrm{R}^{2\left(2^{k-1}-1\right) \cdot(2 \ell+N)},
$$

depending only on $T$ having the property that, for any $\mathbf{y}_{1}, \ldots, \mathbf{y}_{n}, \in \mathrm{R}^{\ell}$ and $\mathbf{a}=$ $\left(\mathbf{a}_{1}, \ldots, \mathbf{a}_{2\left(2^{k-1}-1\right)}\right) \in \mathrm{R}^{2\left(2^{k-1}-1\right) \cdot N}$ where each $\mathbf{a}_{i}$ is a standard basis vector in $\mathrm{R}^{N}$, some sub-collection of the sets

$$
\pi_{k-1+2\left(2^{k-1}-1\right) \cdot(2 \ell+N)}^{\leq k-1}\left(\pi_{k-1+2\left(2^{k-1}-1\right) \cdot(2 \ell+N)}^{>k-1}\left(\mathbf{y}_{i_{1}}, \ldots, \mathbf{y}_{\left.i_{2^{2}(2 k}-1\right)}, \mathbf{a}\right) \cap T_{i}\right),
$$

form a cdcd of $\mathrm{R}^{k}$ compatible with the family

$$
\bigcup_{1 \leq i, j \leq n} \bigcup_{1 \leq h \leq N}\left\{B_{h}\left(\mathbf{y}_{i}, \mathbf{y}_{j}\right)\right\} .
$$

For $\mathbf{x} \in \mathrm{R}^{k-1}$, and $\mathbf{y} \in \mathrm{R}^{\ell}$, let

$$
S(\mathbf{x}, \mathbf{y})=\{x \in \mathrm{R} \mid(x, \mathbf{x}, \mathbf{y}) \in T\} .
$$

Now, for $\mathbf{x} \in \mathrm{R}^{k-1}, \mathbf{y}, \mathbf{y}^{\prime} \in \mathrm{R}^{\ell}, S(\mathbf{x}, \mathbf{y}), S\left(\mathbf{x}, \mathbf{y}^{\prime}\right) \subset \mathrm{R}$ and each of them is a union of a finite number of open intervals and points. The sets $S(\mathbf{x}, \mathbf{y}), S\left(\mathbf{x}, \mathbf{y}^{\prime}\right)$ induce a partition of $\mathrm{R}$ into pairwise disjoint subsets,

$$
V_{1}\left(\mathbf{x}, \mathbf{y}, \mathbf{y}^{\prime}\right), V_{2}\left(\mathbf{x}, \mathbf{y}, \mathbf{y}^{\prime}\right), \ldots,
$$

where for $i \geq 0$, each $V_{2 i+1}\left(\mathbf{x}, \mathbf{y}, \mathbf{y}^{\prime}\right)$ is a maximal open interval contained in one of

$$
\begin{gathered}
S(\mathbf{x}, \mathbf{y}) \cap S\left(\mathbf{x}, \mathbf{y}^{\prime}\right), S(\mathbf{x}, \mathbf{y})^{c} \cap S\left(\mathbf{x}, \mathbf{y}^{\prime}\right), \\
S(\mathbf{x}, \mathbf{y}) \cap S\left(\mathbf{x}, \mathbf{y}^{\prime}\right)^{c}, S(\mathbf{x}, \mathbf{y})^{c} \cap S\left(\mathbf{x}, \mathbf{y}^{\prime}\right)^{c},
\end{gathered}
$$

and $V_{2 i}\left(\mathbf{x}, \mathbf{y}, \mathbf{y}^{\prime}\right)$ is the right end-point of the interval $V_{2 i-1}\left(\mathbf{x}, \mathbf{y}, \mathbf{y}^{\prime}\right)$. We let $\mathcal{V}\left(\mathbf{x}, \mathbf{y}, \mathbf{y}^{\prime}\right)$ denote the ordered sequence,

$$
\left(V_{1}\left(\mathbf{x}, \mathbf{y}, \mathbf{y}^{\prime}\right), V_{2}\left(\mathbf{x}, \mathbf{y}, \mathbf{y}^{\prime}\right), \ldots, V_{M}\left(\mathbf{x}, \mathbf{y}, \mathbf{y}^{\prime}\right)\right)
$$

where $M$ is a uniform upper bound on $|\mathcal{V}|$ depending on $T$, and with the understanding that $V_{i}\left(\mathbf{x}, \mathbf{y}, \mathbf{y}^{\prime}\right)$ can be empty for all $i \geq i_{0}$ for some $0 \leq i_{0} \leq M$. It is clear that the sets,

$$
V_{i}=\left\{\left(V_{i}\left(\mathbf{x}, \mathbf{y}, \mathbf{y}^{\prime}\right), \mathbf{x}, \mathbf{y}, \mathbf{y}^{\prime}\right) \mid \mathbf{x} \in \mathrm{R}^{k-1}, \mathbf{y}, \mathbf{y}^{\prime} \in \mathrm{R}^{\ell}\right\}
$$


are definable and depend only on $T$.

For each $T_{j}^{\prime} \subset \mathrm{R}^{k-1} \times \mathrm{R}^{2\left(2^{k-1}-1\right) \cdot(2 \ell+N)}, j \in J, 1 \leq h \leq M$, and $\mathbf{a}=\left(\mathbf{a}_{1}, \ldots, \mathbf{a}_{2^{k}-2}\right)$, where each $\mathbf{a}_{i}$ is a standard basis vector in $\mathrm{R}^{N}$, let

$$
\begin{gathered}
T_{j, h, \mathbf{a}}^{\prime}=\left\{\left(V_{h}\left(\mathbf{x}, \mathbf{y}_{2^{k+1}-3}, \mathbf{y}_{2^{k+1}-2}\right), \mathbf{x}, \mathbf{y}_{1}, \ldots, \mathbf{y}_{\left(2\left(2^{k}-1\right)\right.}\right) \mid\right. \\
\left.\left(\mathbf{x}, \mathbf{y}_{1}, \ldots, \mathbf{y}_{2^{2}\left(2^{k-1}-1\right)}, \mathbf{a}\right) \in T_{j}^{\prime}\right\} .
\end{gathered}
$$

Let $\left\{T_{i}\right\}_{i \in I}$ be the collection of all possible $T_{j, h, \mathbf{a}}^{\prime}$. It is now easy to verify that the family of sets $\left\{T_{i}\right\}_{i \in I}$ satisfies the conditions of the theorem.

\subsection{Proof of Theorem 2.6}

The proof is very similar to the second proof of Theorem 1.1 in 3 . However, instead of using vertical decomposition as in [3, we use the cylindrical definable cell decomposition given by Theorem 2.5. We repeat it here for the reader's convenience.

Proof of Theorem 2.6. For each $i, 1 \leq i \leq n$, let

$$
A_{i}=\pi_{2 \ell}^{\leq \ell}\left(\pi_{2 \ell}^{>\ell^{-1}}\left(\mathbf{y}_{i}\right) \cap F\right),
$$

and $\mathcal{G}=\left\{A_{i} \mid 1 \leq i \leq n\right\}$. Note that $\mathcal{G}$ is a $\left(R, \pi_{2 \ell}^{\leq \ell}, \pi_{2 \ell}^{>\ell}\right)$-family.

We now use the Clarkson-Shor random sampling technique 22] (using Theorem 2.5 instead of vertical decomposition as in [3). Applying Theorem [2.5 to some sub-family $\mathcal{G}_{0} \subset \mathcal{G}$ of cardinality $r$, we get a decomposition of $\mathrm{R}^{\ell}$ into at most $C r^{2\left(2^{\ell}-1\right)}=r^{O(1)}$ definable cells, each of them defined by at most $2\left(2^{\ell}-1\right)=O(1)$ of the $\mathbf{y}_{i}$ 's. This decomposition satisfies the necessary properties for the existence of $1 / r$-cuttings of size $r^{O(1)}$ [22, pp. 163].

More precisely, let $\tau$ be a cell of the cdcd of $\mathcal{G}_{0}$ and let $G \in \mathcal{G}$. We say that $G$ crosses $\tau$ if $G \cap \tau \neq \emptyset$ and $\tau \not \subset G$. The well-known Cutting Lemma (see [22, Chapter 6, Section 5]) now ensures that we can choose $\mathcal{G}_{0}$ such that each cell of the cdcd of $\mathcal{G}_{0}$ is crossed by no more than $\frac{c_{1} n \log r}{r}$ elements of $\mathcal{G}$, where $c_{1}$ is a constant depending only on $F$.

For each cell $\tau$ of the cdcd of $\mathcal{G}_{0}$, let $\mathcal{G}_{\tau}$ denote the set of elements of $\mathcal{G}$ which cross $\tau$ and let $\mathcal{F}_{\tau}=\mathcal{F} \cap \tau$.

Since the total number of cells in the cdcd of $\mathcal{G}_{0}$ is bounded by $r^{O(1)}$, there must exist a cell $\tau$ such that,

$$
\left|\mathcal{F}_{\tau}\right| \geq \frac{n}{r^{O(1)}} .
$$

Now, every element of $\mathcal{G} \backslash \mathcal{G}_{\tau}$ either fully contains $\tau$ or is disjoint from it.

Setting $\alpha=\frac{1}{r O(1)}$ and $\beta=\frac{1}{2}\left(1-\frac{c_{1} \log r}{r}\right)$ we have that there exists a set $\mathcal{F}^{\prime}=\mathcal{F}_{\tau}$ of cardinality at least $\alpha n$, and a subset $\mathcal{G}^{\prime}$ of cardinality at least $\beta n$ such that either each element of $\mathcal{F}^{\prime}$ is contained in every element of $\mathcal{G}^{\prime}$, or no element of $\mathcal{F}^{\prime}$ is contained in any element of $\mathcal{G}^{\prime}$.

The proof is complete by taking $\mathcal{F}_{1}=\mathcal{F}^{\prime}$, and $\mathcal{F}_{2}=\left\{\mathbf{y}_{i} \mid A_{i} \in \mathcal{G}^{\prime}\right\}$ and choosing $r$ so as to $\operatorname{maximize} \varepsilon=\min (\alpha, \beta)$.

Proof of Corollary2.7. For $1 \leq i \leq n$, let $\mathbf{y}_{i} \in \mathrm{R}^{\ell}$ be such that $S_{i}=T_{\mathbf{y}_{i}}$. Let $F \subset \mathrm{R}^{\ell} \times \mathrm{R}^{\ell}$ be the closed definable set defined by

$$
F=\left\{\left(\mathbf{z}_{1}, \mathbf{z}_{2}\right) \mid \mathbf{z}_{1}, \mathbf{z}_{2} \in \mathrm{R}^{\ell}, T_{\mathbf{z}_{1}} \cap T_{\mathbf{z}_{2}} \neq \emptyset\right\} .
$$

Clearly, $F$ is completely determined by $T$. Now apply Theorem 2.6 .

\section{Conclusion and open problems}

In this paper we have proved bounds on the combinatorial and topological complexities of arrangements of sets belonging to some fixed definable family in an o-minimal structure, in 
terms of the number of sets in the arrangement. These results generalize known results in the case when the sets in the arrangements are semi-algebraic sets and of constant description complexity. We also extended a Ramsey-type theorem due to Alon et al. [3], originally proved for semi-algebraic sets of fixed description complexity to the more general setting of o-minimal geometry.

There are many other sophisticated results on the combinatorial complexity of sub-structures of arrangements which have been proved in the semi-algebraic case. Usually there are some extra assumptions about general position in these results. For instance, it was shown in 7 that the complexity of a single cell in an arrangement of $n$ semi-algebraic hyper-surface patches in $\mathrm{R}^{k}$, which are in general position and have constant description complexity, is bounded by $O\left(n^{k-1+\varepsilon}\right)$. Does this bound also hold for $\left(T, \pi_{1}, \pi_{2}\right)$-families ? It would be interesting to know if all or most results in the computational geometry literature relating to arrangements of sets of constant description complexity, do in fact extend to the more general setting introduced in this paper. It would also be interesting to to find proofs of existing bounds using the kind of homological methods used in this paper. Doing so might remove extraneous assumptions on general positions in several results and possibly even lead to tighter bounds.

Acknowledgments The author thanks an anonymous referee for several helpful remarks that helped to substantially improve the paper.

\section{References}

1. Pankaj K. Agarwal and Jeff Erickson, Geometric range searching and its relatives, Advances in discrete and computational geometry (South Hadley, MA, 1996), Contemp. Math., vol. 223, Amer. Math. Soc. Providence, RI, 1999, pp. 1-56. MR 1661376 (99m:68203)

2. Pankaj K. Agarwal and Micha Sharir, Arrangements and their applications, Handbook of computational geometry (J. Urrutia J.R. Sack, ed.), North-Holland, Amsterdam, 2000, pp. 49-119. MR 1746675

3. Noga Alon, János Pach, Rom Pinchasi, Radoš Radoičić, and Micha Sharir, Crossing patterns of semialgebraic sets, J. Combin. Theory Ser. A 111 (2005), no. 2, 310-326. MR 2156215 (2006k:14108)

4. S. Basu, R. Pollack, and M.-F. Roy, Betti number bounds, applications and algorithms, Current Trends in Combinatorial and Computational Geometry: Papers from the Special Program at MSRI, MSRI Publications, vol. 52, Cambridge University Press, 2005, pp. 87-97.

5. - Algorithms in real algebraic geometry, Algorithms and Computation in Mathematics, vol. 10, Springer-Verlag, Berlin, 2006 (second edition). MR 1998147 (2004g:14064)

6. - Computing the first Betti number of a semi-algebraic set, Found. Comput. Math. 8 (2008), no. 1, $97-136$.

7. Saugata Basu, The combinatorial and topological complexity of a single cell, Discrete Comput. Geom. 29 (2003), no. 1, 41-59. MR 1946793 (2003i:52029)

8. 30 (2003), no. 1, 65-85, ACM Symposium on Computational Geometry (Medford, MA, 2001). MR 1991587 (2004f:14082)

9. Saugata Basu, Richard Pollack, and Marie-Françoise Roy, On the Betti numbers of sign conditions, Proc. Amer. Math. Soc. 133 (2005), no. 4, 965-974 (electronic). MR 2117195 (2006a:14096)

10. A. Bjorner, M. L. Wachs, and V. Welker, Poset fiber theorems, Transactions of the American Mathematical Society 357 (2004), no. 5, 1877-1899.

11. J. Bochnak, M. Coste, and M.-F. Roy, Géométrie algébrique réelle, Ergebnisse der Mathematik und ihrer Grenzgebiete (3) [Results in Mathematics and Related Areas (3)], vol. 12, Springer-Verlag, Berlin, 1987. MR 949442 (90b:14030)

12. M. Coste, An introduction to o-minimal geometry, Istituti Editoriali e Poligrafici Internazionali, Pisa, 2000, Dip. Mat. Univ. Pisa, Dottorato di Ricerca in Matematica.

13. J. H. Davenport and J. Heintz, Real quantifier elimination is doubly exponential, Journal of Symbolic Computation 5 (1988), no. 1/2, 29-35.

14. Mário J. Edmundo and Nicholas J. Peatfield, o-minimal Čech cohomology, Q. J. Math. 59 (2008), no. 2, 213-220. MR 2428077

15. Mário J. Edmundo and Arthur Woerheide, Comparison theorems for o-minimal singular (co)homology, Trans. Amer. Math. Soc. 360 (2008), no. 9, 4889-4912. MR 2403708

16. A. Gabrielov and N. Vorobjov, Complexity of cylindrical decompositions of sub-pfaffian sets, J. Pure and Applied Algebra 164 (2001), 179-197.

17. - Approximation of definable sets by compact families and upper bounds on homotopy and homology, preprint at arXiv:math.AG/0710.3028v1, 2007. 
Page 24 of 24 COMBINATORIAL COMPLEXITY IN O-MINIMAL GEOMETRY

18. A. Gabrielov, N. Vorobjov, and T. Zell, Betti numbers of semialgebraic and sub-Pfaffian sets, J. London Math. Soc. (2) 69 (2004), no. 1, 27-43. MR 2025325 (2004k:14105)

19. Andrei Gabrielov, Complements of subanalytic sets and existential formulas for analytic functions, Invent. Math. 125 (1996), no. 1, 1-12. MR 1389958 (97h:32007)

20. Andrei Gabrielov and Nicolai Vorobjov, Complexity of computations with Pfaffian and Noetherian functions, Normal forms, bifurcations and finiteness problems in differential equations, NATO Sci. Ser. II Math. Phys. Chem., vol. 137, Kluwer Acad. Publ., Dordrecht, 2004, pp. 211-250. MR 2083248 (2006b:14104)

21. ㄴ. Betti numbers of semialgebraic sets defined by quantifier-free formulae, Discrete Comput. Geom. 33 (2005), no. 3, 395-401. MR 2121987 (2005i:14075)

22. Jiří Matoušek, Lectures on discrete geometry, Graduate Texts in Mathematics, vol. 212, Springer-Verlag, New York, 2002. MR 1899299 (2003f:52011)

23. _ Using the Borsuk-Ulam theorem, Universitext, Springer-Verlag, Berlin, 2003, Lectures on topological methods in combinatorics and geometry, Written in cooperation with Anders Björner and Günter M. Ziegler. MR 1988723 (2004i:55001)

24. J. Milnor, On the Betti numbers of real varieties, Proc. Amer. Math. Soc. 15 (1964), 275-280. MR 0161339 $(28$ \# 4547$)$

25. I. G. Petrovskiĭ and O. A. Oleunnik, On the topology of real algebraic surfaces, Izvestiya Akad. Nauk SSSR. Ser. Mat. 13 (1949), 389-402. MR 0034600 (11,613h)

26. A. Pillay and C. Steinhorn, Definable sets in ordered structures. I., Trans. Amer. Math. Soc. 295 (1986), no. 2, 565-592. MR 0833697 (88b:03050a)

27. $\ldots$, Definable sets in ordered structures. III., Trans. Amer. Math. Soc. 309 (1988), no. 2, 469-576. MR 0943306 (89i:03059)

28. J.-P. Rolin, P. Speissegger, and A. J. Wilkie, Quasianalytic Denjoy-Carleman classes and o-minimality, J. Amer. Math. Soc. 16 (2003), no. 4, 751-777 (electronic). MR 1992825 (2004g:14065)

29. René Thom, Sur l'homologie des variétés algébriques réelles, Differential and Combinatorial Topology (A Symposium in Honor of Marston Morse), Princeton Univ. Press, Princeton, N.J., 1965, pp. 255-265. MR 0200942 (34 \#828)

30. Lou van den Dries, Tame topology and o-minimal structures, London Mathematical Society Lecture Note Series, vol. 248, Cambridge University Press, Cambridge, 1998. MR 1633348 (99j:03001)

31. Lou van den Dries and Chris Miller, Geometric categories and o-minimal structures, Duke Math. J. 84 (1996), no. 2, 497-540. MR 1404337 (97i:32008)

32. Lou van den Dries and Patrick Speissegger, The real field with convergent generalized power series, Trans. Amer. Math. Soc. 350 (1998), no. 11, 4377-4421. MR 1458313 (99a:03036)

33. The field of reals with multisummable series and the exponential function, Proc. London Math Soc. (3) 81 (2000), no. 3, 513-565. MR 1781147 (2002k:03057)

34. A. J. Wilkie, Model completeness results for expansions of the ordered field of real numbers by restricted Pfaffian functions and the exponential function, J. Amer. Math. Soc. 9 (1996), no. 4, 1051-1094. MR 1398816 (98j:03052)

35. _ A theorem of the complement and some new o-minimal structures, Selecta Math. (N.S.) 5 (1999), no. 4, 397-421. MR 1740677 (2001c:03071)

\author{
Saugata Basu \\ Department of Mathematics \\ Purdue University \\ West Lafayette, IN 47906 \\ USA
}

sbasu@math.purdue.edu 